ESAIM: COCV 28 (2022) 16

https://doi.org/10.1051/cocv/2022009
ESAIM: Control, Optimisation and Calculus of Variations

www.esaim-cocv.org

\title{
STABILIZATION AND APPROXIMATE NULL-CONTROLLABILITY FOR A LARGE CLASS OF DIFFUSIVE EQUATIONS FROM THICK CONTROL SUPPORTS
}

\author{
Paul Alphonse ${ }^{1, *} \odot$ and JÉRÉmy Martin²®
}

\begin{abstract}
We prove that the thickness property is a necessary and sufficient geometric condition that ensures the (rapid) stabilization or the approximate null-controllability with uniform cost of a large class of evolution equations posed on the whole space $\mathbb{R}^{n}$. These equations are associated with operators of the form $F\left(\left|D_{x}\right|\right)$, the function $F:[0,+\infty) \rightarrow \mathbb{R}$ being continuous and bounded from below. We also provide explicit feedbacks and constants associated with these stabilization properties. The notion of thickness is known to be a necessary and sufficient condition for the exact null-controllability of the fractional heat equations associated with the functions $F(t)=t^{2 s}$ in the case $s>1 / 2$. Our results apply in particular for this class of equations, but also for the half heat equation associated with the function $F(t)=t$, which is the most diffusive fractional heat equation for which exact null-controllability is known to fail from general thick control supports.
\end{abstract}

Mathematics Subject Classification. 93D15, 93B05, 35R11, 26E10.

Received July 5, 2021. Accepted January 29, 2022.

\section{INTRODUCTION}

This paper is devoted to investigate the stabilization and approximate null-controllability for control systems of the following form:

$$
\left\{\begin{array}{l}
\partial_{t} f(t, x)+F\left(\left|D_{x}\right|\right) f(t, x)=h(t, x) \mathbb{1}_{\omega}(x), \quad t>0, x \in \mathbb{R}^{n}, \\
f(0, \cdot)=f_{0} \in L^{2}\left(\mathbb{R}^{n}\right),
\end{array}\right.
$$

where the operator $F\left(\left|D_{x}\right|\right)$ is the Fourier multiplier associated with the symbol $F(|\xi|)$, with $|\cdot|$ the canonical Euclidean norm in $\mathbb{R}^{n}$, the function $F:[0,+\infty) \rightarrow \mathbb{R}$ being continuous and bounded from below, and $\omega \subset \mathbb{R}^{n}$ is a Borel set with positive Lebesgue measure.

The study of the (rapid) stabilization and the (approximate) null-controllability of evolution equations of the form $\left(E_{F}\right)$ has been much addressed recently $[2,3,9,12,16,17,19,21]$. The Schrödinger counterparts of these equations and the same equations posed on bounded domains have also been studied, respectively in [18] and $[15,23]$. In this work, we consider control supports $\omega \subset \mathbb{R}^{n}$ which are thick:

Keywords and phrases: Stabilization, approximate null-controllability, thick sets, quasi-analytic sequences, diffusive equations.

1 Université de Lyon, ENSL, UMPA - UMR 5669, 69364 Lyon, France.

${ }^{2}$ Univ Rennes, CNRS, IRMAR - UMR 6625, 35000 Rennes, France.

* Corresponding author: paul.alphonse@ens-lyon.fr

(C) The authors. Published by EDP Sciences, SMAI 2022

This is an Open Access article distributed under the terms of the Creative Commons Attribution License (https://creativecommons.org/licenses/by/4.0), which permits unrestricted use, distribution, and reproduction in any medium, provided the original work is properly cited. 
Definition 1.1. Given $\gamma \in(0,1)$ and $L>0$, the set $\omega \subset \mathbb{R}^{n}$ is said to be $\gamma$-thick at scale $L$ when it is measurable and satisfies

$$
\forall x \in \mathbb{R}^{n}, \quad \operatorname{Leb}\left(\omega \cap\left(x+[0, L]^{n}\right)\right) \geq \gamma L^{n}
$$

where Leb denotes the Lebesgue measure in $\mathbb{R}^{n}$.

This notion of thickness has appeared to play a key role in the null-controllability theory since the works $[7,22]$, where the authors established that it is a necessary and sufficient geometric condition that ensures the null-controllability of the heat equation posed on $\mathbb{R}^{n}$, which is the equation $\left(E_{F}\right)$ associated with the function $F(t)=t^{2}$. The same phenomenon holds true more generally for the evolution equations associated with fractional Laplacians $(-\Delta)^{s}$ (case where $F(t)=t^{2 s}$ ) in the same setting and when $s>1 / 2$, as proven in [3], and also quite surprisingly for the Schrödinger counterparts of these equations in the one dimensional setting and when $s \geq 1 / 2$, see [18] (Cor. 2.8). It is also known from the works [11, 12] that in the case $0<s \leq 1 / 2$, the fractional heat equation $\left(E_{t^{2 s}}\right)$ is not anymore null-controllable from thick control supports in general. Other classes of degenerate parabolic equations of hypoelliptic type, as evolution equations associated with accretive quadratic operators or (non-autonomous) Ornstein-Uhlenbeck operators, were also proven to be null-controllable from thick control supports, see e.g. $[2,4,5]$. In this work, we prove that a very general class of equations of the form $\left(E_{F}\right)$ is approximately null-controllable with uniform cost from the control support $\omega \subset \mathbb{R}^{n}$ if and only if $\omega$ is a thick set. Our results hold in particular for the half heat equation $\left(E_{t}\right)$ which is not null-controllable from general thick control supports (at least when $n=1$, the case $n \geq 2$ remaining open for the moment), see e.g. [12] (Thm. 2.3) or [16] (Thm. 1.1).

The study of the (rapid) stabilization of the control system $\left(E_{F}\right)$, as for it, has been addressed very recently in the works $[9,17,21]$. It has been proven in [9] (Thm. 1.1) that for all $s>0$, the fractional heat equation $\left(E_{t^{2 s}}\right)$ is exponentially stabilizable from the support $\omega$ if and only if $\omega$ is thick. It is also known from [17] (Exam. 1) that the very same equation $\left(E_{t^{2 s}}\right)$ is rapidly stabilizable from complements of Euclidean balls in $\mathbb{R}^{n}$ when $0<s<1$. In this paper, we establish that the control system $\left(E_{F}\right)$ is exponentially stabilizable from $\omega$ if and only if $\omega$ is a thick set when $\inf F \leq 0$ (in the case $\inf F>0$, the control system $\left(E_{F}\right)$ is stable) and $\lim \inf _{+\infty} F>-\inf F$. Moreover, we provide explicit formulas for the feedbacks $K$ and the constants associated with this stabilization, which allows us to prove that when $\lim _{+\infty} F=+\infty$, the control system $\left(E_{F}\right)$ is rapidly stabilizable from $\omega$ if and only if $\omega$ is thick. In particular, we recover [9] (Thm. 1.1) (with new explicit feedbacks) and we generalize [17] (Exam. 1). We also prove that when $\omega$ is not dense in $\mathbb{R}^{n}$, the equation $\left(E_{F}\right)$ is never rapidly stabilizable in the particular case where $F$ admits a finite limit at $+\infty$.

In a nutshell, our results highlight the importance of the notion of thickness not only in the null-controllability theory, but also for properties of stabilization and approximate null-controllability with uniform cost, as it turns out to be a necessary and sufficient geometric condition ensuring these two properties for a large class of diffusive equations $\left(E_{F}\right)$.

\subsection{Outline of the work}

In Section 2, we present in details the main results contained in this work. Section 3 is devoted to the proofs of the results concerning the stabilization and the rapid stabilization of the control system $\left(E_{F}\right)$. Basic properties of quasi-analytic sequences are presented in Section 4 , which allow to establish the results concerning the approximate null-controllability of the evolution equation $\left(E_{F}\right)$ in Section 5. Finally, Appendix A is an Appendix concerning the proof of an observability result used in Section 5.

\subsection{Notations}

The following notations and conventions will be used all over this work:

1. The canonical Euclidean scalar product of $\mathbb{R}^{n}$ is denoted by $\cdot$ and $|\cdot|$ stands for the associated canonical Euclidean norm. 
2. For all measurable subsets $\omega \subset \mathbb{R}^{n}$, the inner product of $L^{2}(\omega)$ is defined by

$$
\langle u, v\rangle_{L^{2}(\omega)}=\int_{\omega} u(x) \overline{v(x)} \mathrm{d} x, \quad u, v \in L^{2}(\omega),
$$

while $\|\cdot\|_{L^{2}(\omega)}$ stands for the associated norm. Moreover, $\mathcal{L}\left(L^{2}(\omega)\right)$ stands for the set of bounded operators on $L^{2}(\omega)$.

3. For all function $u \in \mathscr{S}\left(\mathbb{R}^{n}\right)$, the Fourier transform of $u$ is denoted $\widehat{u}$ or $\mathscr{F} u$, and defined by

$$
\widehat{u}(\xi)=(\mathscr{F} u)(\xi)=\int_{\mathbb{R}^{n}} e^{-i x \cdot \xi} u(x) \mathrm{d} x, \quad \xi \in \mathbb{R}^{n} .
$$

With this convention, Plancherel's theorem states that

$$
\forall u \in L^{2}\left(\mathbb{R}^{n}\right), \quad\|\widehat{u}\|_{L^{2}\left(\mathbb{R}^{n}\right)}=(2 \pi)^{n / 2}\|u\|_{L^{2}\left(\mathbb{R}^{n}\right)} .
$$

4. We denote by $\nabla_{x}$ the gradient and we set $D_{x}=-i \nabla_{x}$. Moreover, $F\left(\left|D_{x}\right|\right)$ stands for the Fourier multiplier associated with the symbol $F(|\xi|)$ for all continuous function $F:[0,+\infty) \rightarrow \mathbb{R}$.

5. For all measurable subsets $\omega \subset \mathbb{R}^{n}, \mathbb{1}_{\omega}$ stands for the characteristic function of $\omega$.

\section{Statement of the MAIn RESUlts}

This section is devoted to present in details the main results contained in this work. Let us begin by defining precisely the different concepts related to the control system $\left(E_{F}\right)$ we are interested in:

(i) The control system $\left(E_{F}\right)$ is said to be null-controllable from the control support $\omega$ in time $T>0$ when for all $f_{0} \in L^{2}\left(\mathbb{R}^{n}\right)$, there exists a control $h \in L^{2}((0, T) \times \omega)$ such that the mild solution of $\left(E_{F}\right)$ satisfies $f(T, \cdot)=0$.

(ii) The control system $\left(E_{F}\right)$ is said to be approximately null-controllable from the control support $\omega$ in time $T>0$ if for all $\varepsilon>0$ and $f_{0} \in L^{2}\left(\mathbb{R}^{n}\right)$, there exists a control $h \in L^{2}((0, T) \times \omega)$ such that the mild solution of $\left(E_{F}\right)$ satisfies $\|f(T, \cdot)\|_{L^{2}\left(\mathbb{R}^{n}\right)} \leq \varepsilon$.

(iii) The control system $\left(E_{F}\right)$ is said to be approximately null-controllable with uniform cost from the control support $\omega$ in time $T>0$ if for all $\varepsilon>0$, there exists a positive constant $C_{\varepsilon, T}>0$ such that for all $f_{0} \in L^{2}\left(\mathbb{R}^{n}\right)$, there exists a control $h \in L^{2}((0, T) \times \omega)$ such that the mild solution of $\left(E_{F}\right)$ satisfies

$$
\|f(T, \cdot)\|_{L^{2}\left(\mathbb{R}^{n}\right)} \leq \varepsilon\left\|f_{0}\right\|_{L^{2}\left(\mathbb{R}^{n}\right)},
$$

with moreover

$$
\int_{0}^{T}\|h(t, \cdot)\|_{L^{2}(\omega)}^{2} \mathrm{~d} t \leq C_{\varepsilon, T}\left\|f_{0}\right\|_{L^{2}\left(\mathbb{R}^{n}\right)}^{2}
$$

(iv) The control system $\left(E_{F}\right)$ is said to be exponentially stabilizable from the control support $\omega$ at rate $\alpha>0$ if there exist a positive constant $M_{\alpha} \geq 1$ and a feedback $K_{\alpha} \in \mathcal{L}\left(L^{2}\left(\mathbb{R}^{n}\right)\right)$ such that for all $t \geq 0$,

$$
\left\|e^{-t\left(F\left(\left|D_{x}\right|\right)+\mathbb{1}_{\omega} K_{\alpha}\right)}\right\|_{\mathcal{L}\left(L^{2}\left(\mathbb{R}^{n}\right)\right)} \leq M_{\alpha} e^{-\alpha t} .
$$

When the feedback $K_{\alpha}$ can be chosen equal to zero, the control system $\left(E_{F}\right)$ is said to be stable. The existence of the semigroup generated by the operator $F\left(\left|D_{x}\right|\right)+\mathbb{1}_{\omega} K_{\alpha}$ is ensured by the theory of bounded perturbation of semigroups, see e.g. [8] (Thm. III.1.3). 
(v) The control system $\left(E_{F}\right)$ is said to be rapidly stabilizable from the control support $\omega$ if it is exponentially stabilizable from $\omega$ at any rate $\alpha>0$.

\subsection{Stabilization}

First of all, we are interested in tackling stabilization issues for the evolution system $\left(E_{F}\right)$. Let us begin by noticing that when inf $F>0$, we get from Plancherel's theorem that

$$
\forall t \geq 0, \quad\left\|e^{-t F\left(\left|D_{x}\right|\right)}\right\|_{\mathcal{L}\left(L^{2}\left(\mathbb{R}^{n}\right)\right)} \leq e^{-(\inf F) t}
$$

so the control system $\left(E_{F}\right)$ is stable. The interesting case is therefore when $\inf F \leq 0$. In this case, we prove that the thickness of the support $\omega \subset \mathbb{R}^{n}$ is a necessary geometric condition that ensures the stabilization of the equation $\left(E_{F}\right)$, and a sufficient one when assuming in addition that $\liminf _{+\infty} F>|\inf F|$. We also provide explicit feedbacks and quantitative estimates associated with this stabilization.

Theorem 2.1. Let $F:[0,+\infty) \rightarrow \mathbb{R}$ be a continuous function bounded from below and $\omega \subset \mathbb{R}^{n}$ be a measurable set.

(i) If inf $F \leq 0$ and the evolution system $\left(E_{F}\right)$ is exponentially stabilizable from $\omega$, then the set $\omega$ is thick.

(ii) When $\liminf \operatorname{in}_{+\infty} F>\inf F$ and $\omega$ is a thick set, then there exist some positive constants $C=C(\omega) \geq 1$ and $R_{0}=R_{0}(F)>0$ such that for all $R \geq R_{0}$ and $t \geq 0$,

$$
\left\|e^{-t\left(F\left(\left|D_{x}\right|\right)+C e^{C R}\left(\alpha_{R}-\inf F\right) \mathbb{1}_{\omega} K_{R}\right)}\right\|_{\mathcal{L}\left(L^{2}\left(\mathbb{R}^{n}\right)\right)} \leq \sqrt{2} C e^{C R} e^{-\left(\alpha_{R}+\inf F\right) t / 2},
$$

where we set $\alpha_{R}=\inf _{r \geq R} F(r)$ and where $K_{R}$ stands for the following orthogonal projection

$$
K_{R}: L^{2}\left(\mathbb{R}^{n}\right) \rightarrow\left\{f \in L^{2}\left(\mathbb{R}^{n}\right): \operatorname{Supp} \widehat{f} \subset \overline{B(0, R)}\right\},
$$

with $\overline{B(0, R)}$ the closed Euclidean ball centered in 0 with radius $R>0$.

(iii) When $\lim \inf _{+\infty} F>|\inf F|$ and $\omega$ is a thick set, then the evolution system $\left(E_{F}\right)$ is exponentially stabilizable from $\omega$.

Let us check that the assertion (iii) in Theorem 2.1 is a straightforward consequence of the assertion (ii) in the same result. Obviously, the assumption $\liminf _{+\infty} F>|\inf F|$ is read as $\liminf \operatorname{in}_{+\infty} F>\inf F$ and $\liminf _{+\infty} F>$ $-\inf F$. On the one hand, the assumption $\lim \inf _{+\infty} F>\inf F$ implies that the estimates (2.2) hold according to the assertion (ii). On the other hand, assuming that $\liminf _{+\infty} F>-\inf F$, we get that $\alpha_{R}+\inf F>0$ when $R \gg 1$ is large enough. The estimates $(2.2)$ then imply that the evolution equation $\left(E_{F}\right)$ is exponentially stabilizable, according to the definition (2.1) presented in the beginning of this section.

By gathering the results stated in the assertions $(i)$ and $(i i i)$ in Theorem 2.1, we directly obtain the following corollary:

Corollary 2.2. Let $F:[0,+\infty) \rightarrow \mathbb{R}$ be a continuous function bounded from below satisfying inf $F \leq 0$ and $\lim \inf _{+\infty} F>-\inf F$, and $\omega \subset \mathbb{R}^{n}$ be a measurable set. The evolution system $\left(E_{F}\right)$ is exponentially stabilizable from $\omega$ if and only if $\omega$ is thick.

It is a very interesting issue to know whether the control system $\left(E_{F}\right)$ is exponentially stabilizable when $\inf F \leq 0$ and $\liminf +\infty F \leq-\inf F$. We shall not tackle such a question in this work.

As a consequence of the quantitative stabilization estimates (2.2), we directly obtain the following result concerning the rapid stabilization of the evolution system $\left(E_{F}\right)$ under the assumption $\lim _{+\infty} F=+\infty$, by applying Theorem 2.1 to the function $F-\inf F$. 
Corollary 2.3. Let $F:[0,+\infty) \rightarrow \mathbb{R}$ be a continuous function bounded from below satisfying $\lim _{+\infty} F=+\infty$, and $\omega \subset \mathbb{R}^{n}$ be a measurable set. The evolution system $\left(E_{F}\right)$ is rapidly stabilizable from $\omega$ if and only if $\omega$ is thick.

Example 2.4. For all positive real numbers $s>0$, let us consider the function $F_{s}:[0,+\infty) \rightarrow[0,+\infty)$ defined for all $t \geq 0$ by $F_{s}(t)=t^{2 s}$. We also consider $\omega \subset \mathbb{R}^{n}$ a measurable set with positive Lebesgue measure. It follows from Corollaries 2.2 and 2.3 that the associated control system $\left(E_{F_{s}}\right)$ is exponentially stabilizable from the control support $\omega$ if and only if it is rapidly stabilizable from $\omega$ if and only if $\omega$ is a thick set. Moreover, we deduce from Theorem 2.1 that when $\omega$ is thick, there exist positive constants $C \geq 1$ and $R_{0}>0$ such that for all $R \geq R_{0}$ and $t \geq 0$,

$$
\left\|e^{-t\left((-\Delta)^{s}+C e^{C R} R^{2 s} \mathbb{1}_{\omega} K_{R}\right)}\right\|_{\mathcal{L}\left(L^{2}\left(\mathbb{R}^{n}\right)\right)} \leq \sqrt{2} C e^{C R} e^{-R^{2 s} t / 2},
$$

where $K_{R}$ stands for the following orthogonal projection

$$
K_{R}: L^{2}\left(\mathbb{R}^{n}\right) \rightarrow\left\{f \in L^{2}\left(\mathbb{R}^{n}\right): \operatorname{Supp} \widehat{f} \subset \overline{B(0, R)}\right\} .
$$

These explicit stabilization estimates allow to recover [9] (Thm. 1.1) and also to generalize [17] (Exam. 1).

In the case where $\lim _{+\infty} F<+\infty$, we only provide a necessary condition for the control system $\left(E_{F}\right)$ to be rapidly stabilizable. The following result implies in particular that when the function $F$ has a finite limit in $+\infty$ and when the support $\omega \subset \mathbb{R}^{n}$ is not dense in $\mathbb{R}^{n}$, then the equation $\left(E_{F}\right)$ is not rapidly stabilizable from $\omega$.

Proposition 2.5. Let $F:[0,+\infty) \rightarrow \mathbb{R}$ be a continuous function bounded from below and $\omega \subset \mathbb{R}^{n}$ be a measurable set which is not dense in $\mathbb{R}^{n}$. We assume that $\lim _{+\infty} F$ exists and is a non-negative real number (the function $F$ is therefore bounded). Then, if $\alpha>\sup F$, the equation $\left(E_{F}\right)$ is not exponentially stabilizable from $\omega$ at rate $\alpha$.

\subsection{Cost-uniform approximate null-controllability}

In the second part of this work, we study the cost-uniform approximate null-controllability of the equations $\left(E_{F}\right)$. We will not address this question for general continuous functions $F$ bounded from below, but only for the ones generating a quasi-analytic sequence. Let us precisely define this class of functions. Associated with the function $F$ is the following log-convex sequence $\mathcal{M}^{F}$ whose elements $M_{k}^{F}$, assumed to be positive real numbers, are defined by

$$
0<M_{k}^{F}=\sup _{r \geq 0} r^{k} e^{-F(r)}<+\infty, \quad k \geq 0
$$

We say that the sequence $\mathcal{M}^{F}$ is quasi-analytic when, for all real numbers $a<b$, the associated Denjoy-Carleman class

$$
\mathcal{C}_{\mathcal{M}^{F}}([a, b])=\left\{f \in \mathcal{C}^{\infty}([a, b], \mathbb{C}): \forall k \geq 0, \forall x \in[a, b],\left|f^{(k)}(x)\right| \leq M_{k}^{F}\right\},
$$

is quasi-analytic, meaning that any function $f$ in this class satisfying

$$
\exists x_{0} \in[a, b], \forall k \geq 0, \quad f^{(k)}\left(x_{0}\right)=0,
$$

is identically equal to zero. We refer to Section 4 where the notion of quasi-analytic sequence is discussed, and where Denjoy-Carleman's theorem is presented, giving a useful characterization of such sequences.

When the function $F$ generates a quasi-analytic sequence $\mathcal{M}^{F}$ of positive real numbers, the solutions of the homogeneous counterpart of the equation $\left(E_{F}\right)$ belong to quasi-analytic classes of functions (see Sect. 5.1 for 
more details). By taking advantage of this quasi-analytic regularity, we prove that the notion of thickness is a necessary and sufficient geometric condition that ensures the cost-uniform approximate null-controllability of the evolution equations $\left(E_{F}\right)$ in any positive time.

Theorem 2.6. Let $F:[0,+\infty) \rightarrow \mathbb{R}$ be a continuous function bounded from below and $\omega \subset \mathbb{R}^{n}$ be a measurable set. We assume that the sequence $\mathcal{M}^{F}$ associated with the function $F$ defined in $(2.3)$ is a quasi-analytic sequence of positive real numbers. Then, for all positive time $T>0$, the diffusive equation $\left(E_{F}\right)$ is cost-uniformly approximately null-controllable from the control support $\omega$ in time $T$ if and only if $\omega$ is thick.

The necessary part of Theorem 2.6 is a consequence of the fact that the cost-uniform approximate nullcontrollability implies rapid stabilization, see Proposition 5.3, and is therefore a consequence of Corollary 2.3 (notice that the assumption on $F$ in Theorem 2.6 implies in particular that $\lim _{+\infty} F=+\infty$ ).

Let us now present explicit examples of functions $F$ generating quasi-analytic sequences $\mathcal{M}^{F}$ and for which Theorem 2.6 therefore applies.

Example 2.7. Let us assume that the non-negative continuous function $F:[0,+\infty) \rightarrow[0,+\infty)$ satisfies $\Theta \leq F$, where the weight $\Theta:[0,+\infty) \rightarrow[0,+\infty)$ verifies the following properties:

(i) $\Theta(0)=0$ and $\Theta$ is non-decreasing with $\lim _{+\infty} \Theta=+\infty$,

(ii) $\Theta$ is lower-semicontinuous and $t \in \mathbb{R} \mapsto \Theta\left(e^{t}\right)$ is convex,

$$
\int_{0}^{+\infty} \frac{\Theta(t)}{1+t^{2}} \mathrm{~d} t=+\infty
$$

It follows from the work [10] (see also Prop. 4.6 in the present work) that the sequence $\mathcal{M}^{\Theta}$ associated with the weight $\Theta$, defined in (2.3), is quasi-analytic. Moreover, we have $\mathcal{M}^{F} \leq \mathcal{M}^{\Theta}$, since $\Theta \leq F$, and Lemma 4.4 implies that the sequence $\mathcal{M}^{F}$ is also quasi-analytic. We deduce that for all Borel set $\omega \subset \mathbb{R}^{n}$ and all positive time $T>0$, the equation $\left(E_{F}\right)$ is cost-uniformly approximately null-controllable from the set $\omega$ in time $T$ if and only if $\omega$ is thick. A relevant particular example is when $F(t)=\Theta(t)=t$. Indeed, the associated evolution equation is the half heat equation $\left(E_{t}\right)$ posed on the whole space associated with the operator $\sqrt{-\Delta}$, known to be not null-controllable from any non dense control support $\omega$ (at least when $n=1$ ), see [12] (Thm. 2.3) or [16] (Thm. 1.1). This evolution equation is then a relevant example where the thick condition fails to be sufficient for the (strong) null-controllability but appears to be necessary and sufficient for the cost-uniform approximate null-controllability.

Actually, we are able to derive cost-uniform approximate null-controllability results for much less diffusive equations than the half heat equation $\left(E_{t}\right)$, as illustrated in the two following examples.

Example 2.8. Let $s \geq 1,0 \leq \delta \leq 1$ be non-negative real numbers and $F_{s, \delta}:[0,+\infty) \rightarrow[0,+\infty)$ be the non-negative continuous function defined for all $t \geq 0$ by

$$
F_{s, \delta}(t)=\frac{t^{s}}{\log ^{\delta}(e+t)}
$$

We check in Corollary 4.8 that the associated sequence $\mathcal{M}^{F_{s, \delta}}$ defined in (2.3) is a quasi-analytic sequence of positive real numbers. Therefore, for all Borel set $\omega \subset \mathbb{R}^{n}$ and all positive time $T>0$, the equation $\left(E_{F_{s, \delta}}\right)$ is cost-uniformly approximately null-controllable from the set $\omega$ in time $T$ if and only if $\omega$ is thick. 
Example 2.9. Let $p \geq 1$ be a positive integer and $F_{p}:[0,+\infty) \rightarrow[0,+\infty)$ be the non-negative continuous function defined for all $t \geq 0$ by

$$
F_{p}(t)=\frac{t}{g(t)(g \circ g)(t) \ldots g^{\circ p}(t)}, \quad \text { where } \quad g(t)=\log (e+t)
$$

with $g^{\circ p}=g \circ \ldots \circ g$ ( $p$ compositions). We check in Proposition 4.7 that the associated sequence $\mathcal{M}^{F_{p}}$ defined in (2.3) is quasi-analytic. As a consequence, for all Borel set $\omega \subset \mathbb{R}^{n}$ and all positive time $T>0$, the equation $\left(E_{F_{p}}\right)$ is cost-uniformly approximately null-controllable from the set $\omega$ in time $T$ if and only if $\omega$ is thick.

Regarding the weaker notion of approximate null-controllability presented at the beginning of this section, the geometry of the allowed control support is much simpler. Indeed, the following proposition ensures that the control system $\left(E_{F}\right)$ is approximately null-controllable in any positive time $T>0$ and from any measurable set $\omega \subset \mathbb{R}^{n}$ with positive Lebesgue measure when $F$ generates a log-convex quasi-analytic sequence $\mathcal{M}^{F}$.

Proposition 2.10. Let $F:[0,+\infty) \rightarrow \mathbb{R}$ be a continuous function bounded from below and $\omega \subset \mathbb{R}^{n}$ be a measurable set with positive Lebesgue measure. If the sequence $\mathcal{M}^{F}$ associated with the function $F$, defined in (2.3), is a quasi-analytic sequence of positive real numbers, then for all positive time $T>0$, the diffusive equation $\left(E_{F}\right)$ is approximately null-controllable from the support control $\omega$ in time $T$.

In particular, diffusive equations discussed in Examples 2.7, 2.8 and 2.9 are approximately null-controllable in any positive time $T>0$ from any measurable subset $\omega \subset \mathbb{R}^{n}$ satisfying $\operatorname{Leb}(\omega)>0$.

\section{3. (RAPid) Stabilization of Diffusive Equations}

The aim of this section is to prove Theorem 2.1 and Proposition 2.5 concerning the stabilization and the rapid stabilization properties of the following general control system

$$
\left\{\begin{array}{l}
\partial_{t} f(t, x)+F\left(\left|D_{x}\right|\right) f(t, x)=h(t, x) \mathbb{1}_{\omega}(x), \quad t>0, x \in \mathbb{R}^{n}, \\
f(0, \cdot)=f_{0} \in L^{2}\left(\mathbb{R}^{n}\right),
\end{array}\right.
$$

where $F:[0,+\infty) \rightarrow \mathbb{R}$ is a continuous function bounded from below and $\omega \subset \mathbb{R}^{n}$ is a measurable set.

\subsection{Proof of Theorem 2.1: assertion $(i)$}

First of all, let us assume that the equation $\left(E_{F}\right)$ is exponentially stabilizable from the set $\omega$, with the additional assumption that $\inf F \leq 0$. We aim at proving that the control support $\omega$ is then thick. To that end, we will use the following nice characterizations of exponential stabilization in terms of observability estimates, taken from the work [21]:

Theorem 3.1 (Theorem 1 in [21]). The following assertions are equivalent:

(i) The evolution system $\left(E_{F}\right)$ is exponentially stabilizable from $\omega$.

(ii) For all $\varepsilon \in(0,1)$, there exist $T>0$ and $C>0$ such that for all $g \in L^{2}\left(\mathbb{R}^{n}\right)$,

$$
\left\|e^{-T F\left(\left|D_{x}\right|\right)} g\right\|_{L^{2}\left(\mathbb{R}^{n}\right)}^{2} \leq C \int_{0}^{T}\left\|e^{-t F\left(\left|D_{x}\right|\right)} g\right\|_{L^{2}(\omega)}^{2} \mathrm{~d} t+\varepsilon\|g\|_{L^{2}\left(\mathbb{R}^{n}\right)}^{2} .
$$

(iii) There exist $\varepsilon \in(0,1), T>0$ and $C>0$ such that for all $g \in L^{2}\left(\mathbb{R}^{n}\right)$,

$$
\left\|e^{-T F\left(\left|D_{x}\right|\right)} g\right\|_{L^{2}\left(\mathbb{R}^{n}\right)}^{2} \leq C \int_{0}^{T}\left\|e^{-t F\left(\left|D_{x}\right|\right)} g\right\|_{L^{2}(\omega)}^{2} \mathrm{~d} t+\varepsilon\|g\|_{L^{2}\left(\mathbb{R}^{n}\right)}^{2} .
$$


According to the above theorem, assuming that the equation $\left(E_{F}\right)$ is exponentially stabilizable from $\omega$ is equivalent to assuming that there exist $\varepsilon \in(0,1), T>0$ and $C>0$ such that for all $g \in L^{2}\left(\mathbb{R}^{n}\right)$,

$$
\left\|e^{-T F\left(\left|D_{x}\right|\right)} g\right\|_{L^{2}\left(\mathbb{R}^{n}\right)}^{2} \leq C \int_{0}^{T}\left\|e^{-t F\left(\left|D_{x}\right|\right)} g\right\|_{L^{2}(\omega)}^{2} \mathrm{~d} t+\varepsilon\|g\|_{L^{2}\left(\mathbb{R}^{n}\right)}^{2} .
$$

The strategy consists in applying this observability estimate for well-chosen functions $g \in L^{2}\left(\mathbb{R}^{n}\right)$. This approach has especially been used in the works $[2-4,9]$, in which exponential stabilization or null-controllability issues are studied for fractional heat equations or evolution equations associated with (non)-autonomous OrnsteinUhlenbeck operators posed on the whole space $\mathbb{R}^{n}$.

Fixing $x_{0} \in \mathbb{R}^{n}$ and considering $\xi_{0} \in \mathbb{R}^{n}$ together with $l \gg 1$ whose values will be adjusted later, we consider the Gaussian function $g_{l, \xi_{0}}$ defined by

$$
\forall x \in \mathbb{R}^{n}, \quad g_{l, \xi_{0}}(x)=\frac{1}{l^{n}} \exp \left(i x \cdot \xi_{0}-\frac{\left|x-x_{0}\right|^{2}}{2 l^{2}}\right) .
$$

Classical results concerning Fourier transform of Gaussian functions show that

$$
\forall \xi \in \mathbb{R}^{n}, \quad \widehat{g}_{l, \xi_{0}}(\xi)=(2 \pi)^{n / 2} \exp \left(-i x_{0} \cdot\left(\xi-\xi_{0}\right)-\frac{l^{2}\left|\xi-\xi_{0}\right|^{2}}{2}\right) .
$$

On the one hand, it follows from Plancherel's theorem that the left-hand side of the inequality (3.1) applied to the functions $g_{l, \xi_{0}}$ is a positive constant independent of the point $x_{0}$, denoted $\delta_{l, \xi_{0}}>0$ in the following and given by

$$
\begin{aligned}
\delta_{l, \xi_{0}}=\left\|e^{-T F\left(\left|D_{x}\right|\right)} g_{l, \xi_{0}}\right\|_{L^{2}\left(\mathbb{R}^{n}\right)}^{2} & =\int_{\mathbb{R}^{n}}\left|e^{-i x_{0} \cdot\left(\xi-\xi_{0}\right)} e^{-T F(|\xi|)} e^{-l^{2}\left|\xi-\xi_{0}\right|^{2} / 2}\right|^{2} \mathrm{~d} \xi \\
& =\frac{1}{l^{n}} \int_{\mathbb{R}^{n}}\left|e^{-T F\left(\left|\xi / l+\xi_{0}\right|\right)} e^{-|\xi|^{2} / 2}\right|^{2} \mathrm{~d} \xi>0 .
\end{aligned}
$$

On the other hand, we get that the $L^{2}$-norm of the function $g_{l, \xi_{0}}$ also does not depend on the point $x_{0} \in \mathbb{R}^{n}$ and is given by the following Gaussian integral

$$
\left\|g_{l, \xi_{0}}\right\|_{L^{2}\left(\mathbb{R}^{n}\right)}^{2}=\frac{1}{l^{2 n}} \int_{\mathbb{R}^{n}} e^{-|x|^{2} / l^{2}} \mathrm{~d} x=\left(\frac{\pi}{l^{2}}\right)^{n / 2} .
$$

Let us check that the point $\xi_{0} \in \mathbb{R}^{n}$ and the large positive parameter $l \gg 1$ can be adjusted so that $\delta_{l, \xi_{0}}-$ $\varepsilon\left\|g_{l, \xi_{0}}\right\|_{L^{2}\left(\mathbb{R}^{n}\right)}^{2}>0$, that is, by (3.3) and (3.4),

$$
\int_{\mathbb{R}^{n}}\left|e^{-T F\left(\left|\xi / l+\xi_{0}\right|\right)} e^{-|\xi|^{2} / 2}\right|^{2} \mathrm{~d} \xi>\varepsilon \pi^{n / 2}
$$

Since $\varepsilon \in(0,1)$ and the function $F$ satisfies $\inf F \leq 0$, we can assume that the point $\xi_{0} \in \mathbb{R}^{n}$ is chosen in order to satisfy $e^{-2 T F\left(\left|\xi_{0}\right|\right)}>\varepsilon$. Since the function $F$ is bounded from below, the dominated convergence theorem then implies that

$$
\begin{aligned}
\lim _{l \rightarrow+\infty} \int_{\mathbb{R}^{n}}\left|e^{-T F\left(\left|\xi / l+\xi_{0}\right|\right)} e^{-|\xi|^{2} / 2}\right|^{2} \mathrm{~d} \xi & =e^{-2 T F\left(\left|\xi_{0}\right|\right)} \int_{\mathbb{R}^{n}}\left|e^{-|\xi|^{2} / 2}\right|^{2} \mathrm{~d} \xi \\
& =e^{-2 T F\left(\left|\xi_{0}\right|\right)} \pi^{n / 2}>\varepsilon \pi^{n / 2}
\end{aligned}
$$


The parameter $l \gg 1$ can therefore be adjusted so that (3.5) holds. The values of $\xi_{0} \in \mathbb{R}^{n}$ and $l \gg 1$ are now fixed. We therefore deduce from (3.1) and (3.5) that

$$
M_{l, \xi_{0}} \leq C \int_{0}^{T}\left\|e^{-t F\left(\left|D_{x}\right|\right)} g_{l, \xi_{0}}\right\|_{L^{2}(\omega)}^{2} \mathrm{~d} t \quad \text { with } \quad M_{l, \xi_{0}}=\delta_{l, \xi_{0}}-\varepsilon\left\|g_{l, \xi_{0}}\right\|_{L^{2}\left(\mathbb{R}^{n}\right)}^{2}>0 .
$$

Moreover, by introducing $\mathscr{F}_{\xi}^{-1}$ the partial inverse Fourier transform with respect to the variable $\xi \in \mathbb{R}^{n}$ and using (3.2), the right-hand side of this inequality (up to the constant $C$ ) writes as

$$
\begin{aligned}
\int_{0}^{T}\left\|e^{-t F\left(\left|D_{x}\right|\right)} g_{l, \xi_{0}}\right\|_{L^{2}(\omega)}^{2} \mathrm{~d} t & =(2 \pi)^{n} \int_{0}^{T} \int_{\omega}\left|\mathscr{F}_{\xi}^{-1}\left(e^{-i x_{0} \cdot\left(\xi-\xi_{0}\right)} e^{-t F(|\xi|)} e^{-l^{2}\left|\xi-\xi_{0}\right|^{2} / 2}\right)(x)\right|^{2} \mathrm{~d} x \mathrm{~d} t \\
& =(2 \pi)^{n} \int_{0}^{T} \int_{\omega}\left|\mathscr{F}_{\xi}^{-1}\left(e^{-t F(|\xi|)} e^{-l^{2}\left|\xi-\xi_{0}\right|^{2} / 2}\right)\left(x-x_{0}\right)\right|^{2} \mathrm{~d} x \mathrm{~d} t \\
& =(2 \pi)^{n} \int_{0}^{T} \int_{\omega-x_{0}}\left|\mathscr{F}_{\xi}^{-1}\left(e^{-t F(|\xi|)} e^{-l^{2}\left|\xi-\xi_{0}\right|^{2} / 2}\right)(x)\right|^{2} \mathrm{~d} x \mathrm{~d} t
\end{aligned}
$$

Given $r>0$ a positive radius whose value will be chosen later, we split the previous integral in two parts and obtain the following estimate:

$$
\begin{aligned}
& \int_{0}^{T}\left\|e^{-t F\left(\left|D_{x}\right|\right)} g_{l, \xi_{0}}\right\|_{L^{2}(\omega)}^{2} \mathrm{~d} t \\
& \leq(2 \pi)^{n} \int_{0}^{T} \int_{\left(\omega-x_{0}\right) \cap[-r, r]^{n}}\left|\mathscr{F}_{\xi}^{-1}\left(e^{-t F(|\xi|)} e^{-l^{2}\left|\xi-\xi_{0}\right|^{2} / 2}\right)(x)\right|^{2} \mathrm{~d} x \mathrm{~d} t \\
&+(2 \pi)^{n} \int_{0}^{T} \int_{|x|>r}\left|\mathscr{F}_{\xi}^{-1}\left(e^{-t F(|\xi|)} e^{-l^{2}\left|\xi-\xi_{0}\right|^{2} / 2}\right)(x)\right|^{2} \mathrm{~d} x \mathrm{~d} t .
\end{aligned}
$$

Now, we study one by one the two integrals appearing in the right-hand side of (3.7). First, notice that for all $0 \leq t \leq T$,

$$
\begin{aligned}
\left\|\mathscr{F}_{\xi}^{-1}\left(e^{-t F(|\xi|)} e^{-l^{2}\left|\xi-\xi_{0}\right|^{2} / 2}\right)\right\|_{L^{\infty}\left(\mathbb{R}^{n}\right)} & \leq \frac{1}{(2 \pi)^{n}}\left\|e^{-t F(|\xi|)} e^{-l^{2}\left|\xi-\xi_{0}\right|^{2} / 2}\right\|_{L^{1}\left(\mathbb{R}^{n}\right)} \\
& \leq \frac{e^{-T \inf F}}{(2 \pi)^{n}}\left\|e^{-l^{2}\left|\xi-\xi_{0}\right|^{2} / 2}\right\|_{L^{1}\left(\mathbb{R}^{n}\right)} \\
& =\frac{e^{-T \inf F}}{(2 \pi)^{n}}\left(\frac{2 \pi}{l^{2}}\right)^{n / 2}
\end{aligned}
$$

It therefore follows from the invariance by translation of the Lebesgue measure that

$$
\begin{aligned}
(2 \pi)^{n} \int_{0}^{T} \int_{\left(\omega-x_{0}\right)} \cap[-r, r]^{n} & \left|\mathscr{F}_{\xi}^{-1}\left(e^{-t F(|\xi|)} e^{-l^{2}\left|\xi-\xi_{0}\right|^{2} / 2}\right)(x)\right|^{2} \mathrm{~d} x \mathrm{~d} t \\
\leq & \frac{e^{-2 T \inf F}}{l^{2 n}} \int_{0}^{T} \operatorname{Leb}\left(\left(\omega-x_{0}\right) \cap[-r, r]^{n}\right) \mathrm{d} t=\frac{T e^{-2 T \inf F}}{l^{2 n}} \operatorname{Leb}\left(\omega \cap\left(x_{0}+[-r, r]^{n}\right)\right) .
\end{aligned}
$$


In order to control the second integral, we use the dominated convergence theorem which justifies the following convergence

$$
\int_{0}^{T} \int_{|x|>r}\left|\mathscr{F}_{\xi}^{-1}\left(e^{-t F(|\xi|)} e^{-l^{2}\left|\xi-\xi_{0}\right|^{2} / 2}\right)(x)\right|^{2} \mathrm{~d} x \mathrm{~d} t \underset{r \rightarrow+\infty}{\rightarrow} 0
$$

since

$$
\mathscr{F}_{\xi}^{-1}\left(e^{-t F(|\xi|)} e^{-l^{2}\left|\xi-\xi_{0}\right|^{2} / 2}\right) \in L^{2}\left([0, T] \times \mathbb{R}^{n}\right) .
$$

Thus, we can choose the radius $r \gg 1$ large enough so that

$$
(2 \pi)^{n} C \int_{0}^{T} \int_{|x|>r}\left|\mathscr{F}_{\xi}^{-1}\left(e^{-t F(|\xi|)} e^{-l^{2}\left|\xi-\xi_{0}\right|^{2} / 2}\right)(x)\right|^{2} \mathrm{~d} x \mathrm{~d} t \leq \frac{M_{l, \xi_{0}}}{2} .
$$

Gathering (3.6), (3.7), (3.8) and (3.9), we obtain the following estimate

$$
\forall x_{0} \in \mathbb{R}^{n}, \quad \frac{M_{l, \xi_{0}}}{2} \leq \frac{C}{l^{2 n}} T e^{-2 T \inf F} \operatorname{Leb}\left(\omega \cap\left(x_{0}+[-r, r]^{n}\right)\right) .
$$

This proves that the control support $\omega$ is actually a thick set.

\subsection{Proof of Theorem 2.1: assertion (ii)}

In this second subsection, assuming that $\omega$ is a thick set and that $\liminf _{+\infty} F>\inf F$, we establish the estimate (2.2). The strategy consists in constructing an adapted Lyapunov function, inspired by the proof of [23] (Thm. 1.1) in which the author studies the stabilization properties of the heat equation posed on bounded domains of $\mathbb{R}^{n}$.

We consider the function $G=F-\inf F$. Since $\lim _{\inf _{+\infty}} G>0$ by assumption, there exists a positive constant $R_{0}>0$ such that

$$
\forall R \geq R_{0}, \quad \tilde{\alpha}_{R}=\inf _{r \geq R} G(r)>0
$$

Let us fix $R \geq R_{0}$ and consider two positive real numbers $\lambda_{R}, \mu_{R}>0$ to be chosen later. For all initial data $f_{0} \in L^{2}\left(\mathbb{R}^{n}\right)$ and all time $t \geq 0$, we define $f$ as the mild solution on $[0,+\infty)$ of the control system $\left(E_{G}\right)$ with feedback $\lambda_{R} K_{R}$ at time $t$, that is,

$$
\forall t \geq 0, \quad f(t)=e^{-t\left(G\left(\left|D_{x}\right|\right)+\lambda_{R} \mathbb{1}_{\omega} K_{R}\right)} f_{0},
$$

where $K_{R}$ stands for the following orthogonal projection

$$
K_{R}: L^{2}\left(\mathbb{R}^{n}\right) \rightarrow\left\{g \in L^{2}\left(\mathbb{R}^{n}\right): \operatorname{Supp} \widehat{g} \subset \overline{B(0, R)}\right\} .
$$

We want to prove that the two constants $\lambda_{R}$ and $\mu_{R}$ can be adjusted so that the following estimate holds for all $t \geq 0$,

$$
\|f(t)\|_{L^{2}\left(\mathbb{R}^{n}\right)}^{2} \leq \mu_{R} e^{-\tilde{\alpha}_{R} t}\left\|f_{0}\right\|_{L^{2}\left(\mathbb{R}^{n}\right)}^{2} .
$$


To that end, we consider the following Lyapunov function

$$
V(y)=\mu_{R}\left\|K_{R} y\right\|_{L^{2}\left(\mathbb{R}^{n}\right)}^{2}+\left\|\left(1-K_{R}\right) y\right\|_{L^{2}\left(\mathbb{R}^{n}\right)}^{2}, \quad y \in L^{2}\left(\mathbb{R}^{n}\right) .
$$

Let us equip the operator $G\left(\left|D_{x}\right|\right)+\lambda_{R} \mathbb{1}_{\omega} K_{R}$ with the following domain

$$
D_{G}=\left\{u \in L^{2}\left(\mathbb{R}^{n}\right): G\left(\left|D_{x}\right|\right) u \in L^{2}\left(\mathbb{R}^{n}\right)\right\} .
$$

From now, we assume that the Fourier transform of the initial datum $f_{0}$ is compactly supported. As a consequence, $f_{0} \in D_{G}$ and the function $V \circ f$ is of class $C^{1}$ on $(0,+\infty)$, with

$$
\forall t>0, \quad \frac{\mathrm{d}}{\mathrm{d} t} V(f(t))=\mu_{R} \frac{\mathrm{d}}{\mathrm{d} t}\left\|K_{R} f(t)\right\|_{L^{2}\left(\mathbb{R}^{n}\right)}^{2}+\frac{\mathrm{d}}{\mathrm{d} t}\left\|\left(1-K_{R}\right) f(t)\right\|_{L^{2}\left(\mathbb{R}^{n}\right)}^{2} .
$$

We shall need to estimate each term of the right-hand side of this equality. On the one hand, noticing that the operators $G\left(\left|D_{x}\right|\right)$ and $K_{R}$ commute (they are Fourier multipliers), we have that for all $t>0$,

$$
\begin{aligned}
\mu_{R} \frac{\mathrm{d}}{\mathrm{d} t}\left\|K_{R} f(t)\right\|_{L^{2}\left(\mathbb{R}^{n}\right)}^{2}=2 \mu_{R} \operatorname{Re}\left\langle K_{R} f(t)\right. & \left.K_{R} f^{\prime}(t)\right\rangle_{L^{2}\left(\mathbb{R}^{n}\right)} \\
& =-2 \mu_{R}\left\langle K_{R} f(t), G\left(\left|D_{x}\right|\right) K_{R} f(t)\right\rangle_{L^{2}\left(\mathbb{R}^{n}\right)}-2 \lambda_{R} \mu_{R}\left\|K_{R} f(t)\right\|_{L^{2}(\omega)}^{2} .
\end{aligned}
$$

The operator $G\left(\left|D_{x}\right|\right)$ being accretive, we get that for all $t>0$,

$$
\left\langle K_{R} f(t), G\left(\left|D_{x}\right|\right) K_{R} f(t)\right\rangle_{L^{2}\left(\mathbb{R}^{n}\right)} \geq 0,
$$

and as a consequence,

$$
\mu_{R} \frac{\mathrm{d}}{\mathrm{d} t}\left\|K_{R} f(t)\right\|_{L^{2}\left(\mathbb{R}^{n}\right)}^{2} \leq-2 \lambda_{R} \mu_{R}\left\|K_{R} f(t)\right\|_{L^{2}(\omega)}^{2} .
$$

Moreover, O. Kovrijkine established in [14] (Thm. 3) a quantitative version of the Logvinenko-Sereda theorem for thick sets which will allow us to control the right-hand side of the above estimate. Precisely, this result is the following:

Theorem 3.2 (Theorem 3 in [14]). There exists a universal positive constant $C_{n} \geq e$ depending only on the dimension $n \geq 1$ such that for all $\gamma$-thick at scale $L>0$ subset $\omega \subset \mathbb{R}^{n}$,

$$
\forall R>0, \forall f \in L^{2}\left(\mathbb{R}^{n}\right), \operatorname{Supp} \widehat{f} \subset[-R, R]^{n}, \quad\|f\|_{L^{2}\left(\mathbb{R}^{n}\right)} \leq\left(\frac{C_{n}}{\gamma}\right)^{C_{n}(1+L R)}\|f\|_{L^{2}(\omega)} .
$$

We therefore deduce from this theorem and the definition (3.12) of the orthogonal projection $K_{R}$ that there exists a positive constant $C=C(\omega) \geq 1$ only depending on the thick set $\omega$ (and not on the positive real number $R$ ) such that for all $t>0$,

$$
\mu_{R} \frac{\mathrm{d}}{\mathrm{d} t}\left\|K_{R} f(t)\right\|_{L^{2}\left(\mathbb{R}^{n}\right)}^{2} \leq-2 \lambda_{R} \mu_{R} C^{-1} e^{-C R}\left\|K_{R} f(t)\right\|_{L^{2}\left(\mathbb{R}^{n}\right)}^{2} .
$$

On the other hand, recalling that the operators $G\left(\left|D_{x}\right|\right)$ and $K_{R}$ commute, the second term we aim at controlling is given for all $t>0$ by 


$$
\begin{aligned}
& \frac{\mathrm{d}}{\mathrm{d} t}\left\|\left(1-K_{R}\right) f(t)\right\|_{L^{2}\left(\mathbb{R}^{n}\right)}^{2}=2 \operatorname{Re}\left\langle\left(1-K_{R}\right) f(t),\left(1-K_{R}\right) f^{\prime}(t)\right\rangle_{L^{2}\left(\mathbb{R}^{n}\right)} \\
& =-2\left\langle\left(1-K_{R}\right) f(t), G\left(\left|D_{x}\right|\right)\left(1-K_{R}\right) f(t)\right\rangle_{L^{2}\left(\mathbb{R}^{n}\right)}-2 \lambda_{R} \operatorname{Re}\left\langle\left(1-K_{R}\right) f(t),\left(1-K_{R}\right) \mathbb{1}_{\omega} K_{R} f(t)\right\rangle_{L^{2}\left(\mathbb{R}^{n}\right)} .
\end{aligned}
$$

We notice that by definition of the orthogonal projection $K_{R}$, the Fourier transforms of the functions $(1-$ $\left.K_{R}\right) f(t)$ are supported in $\mathbb{R}^{n} \backslash B(0, R)$, which implies that for all $t>0$,

$$
2\left\langle\left(1-K_{R}\right) f(t), G\left(\left|D_{x}\right|\right)\left(1-K_{R}\right) f(t)\right\rangle_{L^{2}\left(\mathbb{R}^{n}\right)} \geq 2 \tilde{\alpha}_{R}\left\|\left(1-K_{R}\right) f(t)\right\|_{L^{2}\left(\mathbb{R}^{n}\right)}^{2} .
$$

By using in addition Cauchy-Schwarz' and Young's inequalities, we obtain

$$
\begin{aligned}
-2 \lambda_{R} \operatorname{Re}\left\langle\left(1-K_{R}\right) f(t),\left(1-K_{R}\right) \mathbb{1}_{\omega} K_{R} f(t)\right\rangle_{L^{2}\left(\mathbb{R}^{n}\right)} & \leq 2 \lambda_{R}\left\|\left(1-K_{R}\right) f(t)\right\|_{L^{2}\left(\mathbb{R}^{n}\right)}\left\|K_{R} f(t)\right\|_{L^{2}\left(\mathbb{R}^{n}\right)} \\
& \leq \frac{\lambda_{R}^{2}}{\tilde{\alpha}_{R}}\left\|K_{R} f(t)\right\|_{L^{2}\left(\mathbb{R}^{n}\right)}^{2}+\tilde{\alpha}_{R}\left\|\left(1-K_{R}\right) f(t)\right\|_{L^{2}\left(\mathbb{R}^{n}\right)}^{2} .
\end{aligned}
$$

Combining the estimates (3.16), (3.17) and (3.18), we obtain that for all $t>0$,

$$
\frac{\mathrm{d}}{\mathrm{d} t}\left\|\left(1-K_{R}\right) f(t)\right\|_{L^{2}\left(\mathbb{R}^{n}\right)}^{2} \leq \frac{\lambda_{R}^{2}}{\tilde{\alpha}_{R}}\left\|K_{R} f(t)\right\|_{L^{2}\left(\mathbb{R}^{n}\right)}^{2}-\tilde{\alpha}_{R}\left\|\left(1-K_{R}\right) f(t)\right\|_{L^{2}\left(\mathbb{R}^{n}\right)}^{2} .
$$

This inequality and (3.15) then imply that for all $t>0$,

$$
\frac{\mathrm{d}}{\mathrm{d} t} V(f(t)) \leq-2\left(\lambda_{R} \mu_{R} C^{-1} e^{-C R}-\frac{\lambda_{R}^{2}}{\tilde{\alpha}_{R}}\right)\left\|K_{R} f(t)\right\|_{L^{2}\left(\mathbb{R}^{n}\right)}^{2}-\tilde{\alpha}_{R}\left\|\left(1-K_{R}\right) f(t)\right\|_{L^{2}\left(\mathbb{R}^{n}\right)}^{2} .
$$

By making the following choices for the constants $\mu_{R}$ and $\lambda_{R}$,

$$
\mu_{R}=2 C^{2} e^{2 C R}, \quad \lambda_{R}=C e^{C R} \tilde{\alpha}_{R},
$$

we get that for all $t>0$,

$$
\frac{\mathrm{d}}{\mathrm{d} t} V(f(t)) \leq-\tilde{\alpha}_{R} V(f(t))
$$

This latest estimate and Grönwall's inequality readily imply that for all $t \geq 0$,

$$
V(f(t)) \leq e^{-\tilde{\alpha}_{R} t} V(f(0)),
$$

and then, by Pythagore's theorem, since $\mu_{R} \geq 1$, we obtain that for all $t \geq 0$,

$$
\|f(t)\|_{L^{2}\left(\mathbb{R}^{n}\right)}^{2} \leq \mu_{R} e^{-\tilde{\alpha}_{R} t}\left\|f_{0}\right\|_{L^{2}\left(\mathbb{R}^{n}\right)}^{2} .
$$

Since the set of functions of $L^{2}\left(\mathbb{R}^{n}\right)$ with compactly supported Fourier transforms is dense in $L^{2}\left(\mathbb{R}^{n}\right)$, and that the evolution operators $e^{-t\left(G\left(\left|D_{x}\right|\right)+\lambda_{R} \mathbb{1}_{\omega} K_{R}\right)}$ are continuous on this space, the above estimate is valid for all $f_{0} \in L^{2}\left(\mathbb{R}^{n}\right)$. The estimate (3.13) therefore holds. Recalling the definitions (3.10) and (3.11) of $\tilde{\alpha}_{R}$ and $f(t)$ respectively, and also recalling that $G=F-\inf F$, we have established that for all $R \geq R_{0}$ and $t \geq 0$,

$$
\left\|e^{-t\left(F\left(\left|D_{x}\right|\right)+C e^{C R}\left(\alpha_{R}-\inf F\right) \mathbb{1}_{\omega} K_{R}\right)}\right\|_{\mathcal{L}\left(L^{2}\left(\mathbb{R}^{n}\right)\right)}^{2} \leq 2 C^{2} e^{2 C R} e^{-\left(\alpha_{R}+\inf F\right) t}
$$


with $\alpha_{R}=\inf _{r \geq R} F(r)$. This ends the proof of assertion $(i i)$ in Theorem 2.1.

\subsection{Proof of Proposition 2.5}

In this last subsection, we prove Proposition 2.5 which provides a negative result for the rapid stabilization of the evolution equation $\left(E_{F}\right)$. We assume that $\omega$ is not dense in $\mathbb{R}^{n}$, and also that $\lim _{+\infty} F$ exists and is a non-negative real number $L \geq 0$. Since the function $F$ is continuous, this implies that $F$ is bounded, i.e. $\sup F<+\infty$. We aim at proving that if $\alpha>\sup F$, then the equation $\left(E_{F}\right)$ is not exponentially stabilizable from $\omega$ at rate $\alpha$. To that end, we will use the following interpretation of exponential stabilization at rate $\alpha>0$ in terms of observability.

Proposition 3.3 (Theorem 1.1 in [17]). If the evolution system $\left(E_{F}\right)$ is exponentially stabilizable from $\omega$ at rate $\alpha>0$, then there exists a positive constant $A_{\alpha}>0$ such that for all $T>0$, there exists a positive constant $C_{\alpha, T}>0$ satisfying that for all $g \in L^{2}\left(\mathbb{R}^{n}\right)$,

$$
\left\|e^{-T F\left(\left|D_{x}\right|\right)} g\right\|_{L^{2}\left(\mathbb{R}^{n}\right)}^{2} \leq C_{\alpha, T} \int_{0}^{T}\left\|e^{-t F\left(\left|D_{x}\right|\right)} g\right\|_{L^{2}(\omega)}^{2} \mathrm{~d} t+A_{\alpha} e^{-2 \alpha T}\|g\|_{L^{2}\left(\mathbb{R}^{n}\right)}^{2}
$$

The proof of Proposition 3.3 is contained in the proof of Theorem 1.1 in [17], although [17] (Thm. 1.1) only states characterizations of complete stabilization. For the sake of completeness, we recall the arguments given by the authors of [17] in Appendix A.

Proceeding by contradiction, we consider $\alpha>\sup F$ and assume that the equation $\left(E_{F}\right)$ is exponentially stabilizable at rate $\alpha$ from $\omega$. According to Proposition 3.3, there exists a positive constant $A_{\alpha}>0$ such that for all $T>0$, there exists a positive constant $C_{\alpha, T}>0$ such that for all $g \in L^{2}\left(\mathbb{R}^{n}\right)$,

$$
\left\|e^{-T F\left(\left|D_{x}\right|\right)} g\right\|_{L^{2}\left(\mathbb{R}^{n}\right)}^{2} \leq C_{\alpha, T} \int_{0}^{T}\left\|e^{-t F\left(\left|D_{x}\right|\right)} g\right\|_{L^{2}(\omega)}^{2} \mathrm{~d} t+A_{\alpha} e^{-2 \alpha T}\|g\|_{L^{2}\left(\mathbb{R}^{n}\right)}^{2} .
$$

Since we get that for all $T>0$ and $g \in L^{2}\left(\mathbb{R}^{n}\right)$,

$$
\left\|e^{-T F\left(\left|D_{x}\right|\right)} g\right\|_{L^{2}\left(\mathbb{R}^{n}\right)} \geq e^{-T \sup F}\|g\|_{L^{2}\left(\mathbb{R}^{n}\right)},
$$

it follows from (3.19) that for all $T>0$ and $g \in L^{2}\left(\mathbb{R}^{n}\right)$,

$$
\|g\|_{L^{2}\left(\mathbb{R}^{n}\right)}^{2} \leq C_{\alpha, T} e^{2 T \sup F} \int_{0}^{T}\left\|e^{-t F\left(\left|D_{x}\right|\right)} g\right\|_{L^{2}(\omega)}^{2} \mathrm{~d} t+A_{\alpha} e^{2(\sup F-\alpha) T}\|g\|_{L^{2}\left(\mathbb{R}^{n}\right)}^{2} .
$$

Notice that $\operatorname{since} \sup F-\alpha<0$, we have $\lim _{T \rightarrow+\infty} e^{2(\sup F-\alpha) T}=0$. Therefore, there exists $T_{0}>0$ such that $A_{\alpha} e^{2(\sup F-\alpha) T_{0}}<1 / 2$. This fact, together with (3.20), imply that for all $g \in L^{2}\left(\mathbb{R}^{n}\right)$,

$$
\|g\|_{L^{2}\left(\mathbb{R}^{n}\right)}^{2} \leq 2 C_{\alpha, T_{0}} e^{2 T_{0} \sup F} \int_{0}^{T_{0}}\left\|e^{-t F\left(\left|D_{x}\right|\right)} g\right\|_{L^{2}(\omega)}^{2} \mathrm{~d} t .
$$

Since $\omega$ is not dense in $\mathbb{R}^{n}$ and that the evolution equation $\left(E_{F}\right)$ is invariant under translations, we can assume that there exists a positive radius $r>0$ such that $B(0, r) \subset \mathbb{R}^{n} \backslash \omega$. Let us fix a non-zero $L^{2}$-function $\psi$ and define the function $g_{h}$ for all $h>0$ by

$$
\forall x \in \mathbb{R}^{n}, \quad g_{h}(x)=\psi\left(\frac{x}{h}\right)
$$


On the one hand, we have that for all $h>0$,

$$
\left\|g_{h}\right\|_{L^{2}\left(\mathbb{R}^{n}\right)}^{2}=h^{n}\|\psi\|_{L^{2}\left(\mathbb{R}^{n}\right)}^{2} .
$$

On the other hand, we get that for all $h>0$,

$$
\begin{aligned}
\int_{0}^{T_{0}}\left\|e^{-t F\left(\left|D_{x}\right|\right)} g_{h}\right\|_{L^{2}(\omega)}^{2} \mathrm{~d} t & \leq \int_{0}^{T_{0}}\left\|e^{-t F\left(\left|D_{x}\right|\right)} g_{h}\right\|_{L^{2}\left(\mathbb{R}^{n} \backslash B(0, r)\right)}^{2} \mathrm{~d} t \\
& =h^{n} \int_{0}^{T_{0}}\left\|e^{-t F\left(\left|D_{x}\right| / h\right)} \psi\right\|_{L^{2}\left(\mathbb{R}^{n} \backslash B(0, r / h)\right)}^{2} \mathrm{~d} t .
\end{aligned}
$$

It follows from (3.21), (3.22) and (3.23) that for all $h>0$,

$$
\|\psi\|_{L^{2}\left(\mathbb{R}^{n}\right)}^{2} \leq 2 C_{\alpha, T_{0}} e^{2 T_{0} \sup F} \int_{0}^{T_{0}}\left\|e^{-t F\left(\left|D_{x}\right| / h\right)} \psi\right\|_{L^{2}\left(\mathbb{R}^{n} \backslash B(0, r / h)\right)}^{2} \mathrm{~d} t .
$$

Moreover, since $L=\lim _{+\infty} F \geq 0$, we get

$$
\begin{aligned}
& \int_{0}^{T_{0}}\left\|e^{-t F\left(\left|D_{x}\right| / h\right)} \psi\right\|_{L^{2}\left(\mathbb{R}^{n} \backslash B(0, r / h)\right)}^{2} \mathrm{~d} t \\
\leq & 2 \int_{0}^{T_{0}}\left\|e^{-t F\left(\left|D_{x}\right| / h\right)} \psi-e^{-t L} \psi\right\|_{L^{2}\left(\mathbb{R}^{n} \backslash B(0, r / h)\right)}^{2} \mathrm{~d} t+2 \int_{0}^{T_{0}}\left\|e^{-t L} \psi\right\|_{L^{2}\left(\mathbb{R}^{n} \backslash B(0, r / h)\right)}^{2} \mathrm{~d} t \\
\leq & 2 \int_{0}^{T_{0}}\left\|e^{-t F\left(\left|D_{x}\right| / h\right)} \psi-e^{-t L} \psi\right\|_{L^{2}\left(\mathbb{R}^{n}\right)}^{2} \mathrm{~d} t+2 T_{0}\|\psi\|_{L^{2}\left(\mathbb{R}^{n} \backslash B(0, r / h)\right)}^{2},
\end{aligned}
$$

and this implies that

$$
\|\psi\|_{L^{2}\left(\mathbb{R}^{n}\right)}^{2} \leq 4 C_{\alpha, T_{0}} e^{2 T_{0} \sup F} \int_{0}^{T_{0}}\left\|e^{-t F\left(\left|D_{x}\right| / h\right)} \psi-e^{-t L} \psi\right\|_{L^{2}\left(\mathbb{R}^{n}\right)}^{2} \mathrm{~d} t+4 C_{\alpha, T_{0}} T_{0} e^{2 T_{0} \sup F}\|\psi\|_{L^{2}\left(\mathbb{R}^{n} \backslash B(0, r / h)\right)}^{2} .
$$

To obtain a contradiction, it remains to check that each term of the right-hand side of the above inequality converges to 0 as $h \rightarrow 0^{+}$. For the first term, we deduce from Plancherel's theorem and the dominated convergence theorem (recall that the function $F$ is bounded from below) that

$$
\int_{0}^{T_{0}}\left\|e^{-t F\left(\left|D_{x}\right| / h\right)} \psi-e^{-t L} \psi\right\|_{L^{2}\left(\mathbb{R}^{n}\right)}^{2} \mathrm{~d} t=\frac{1}{(2 \pi)^{n}} \int_{0}^{T_{0}}\left\|e^{-t F(|\xi| / h)} \widehat{\psi}-e^{-t L} \widehat{\psi}\right\|_{L^{2}\left(\mathbb{R}^{n}\right)}^{2} \mathrm{~d} t \underset{h \rightarrow 0}{\rightarrow} 0 .
$$

For the second one, the dominated convergence theorem readily implies that

$$
\|\psi\|_{L^{2}\left(\mathbb{R}^{n} \backslash B(0, r / h)\right)}^{2} \underset{h \rightarrow 0}{\rightarrow} 0 .
$$

This concludes the proof of Proposition 2.5.

\section{QUASI-ANALYTIC SEQUENCES}

This section is devoted to recall some properties of quasi-analytic sequences. 


\subsection{Notion of quasi-analytic sequence}

Let us first recall that a sequence $\left(M_{k}\right)_{k \geq 0}$ of non-negative real numbers is said to be log-convex when it satisfies

$$
\forall k \geq 1, \quad M_{k}^{2} \leq M_{k+1} M_{k-1}
$$

A relevant example of log-convex sequence is the sequence $\mathcal{M}^{F}$ associated with any continuous function $F$ : $[0,+\infty) \rightarrow \mathbb{R}$ bounded from below. Let us recall that the elements $M_{k}^{F}$ of the sequence $\mathcal{M}^{F}$ are assumed to be positive real numbers and defined for all $k \geq 0$ by

$$
0<M_{k}^{F}=\sup _{r \geq 0} r^{k} e^{-F(r)}<+\infty
$$

Let us also recall that a sequence $\mathcal{M}=\left(M_{k}\right)_{k>0}$ of positive real numbers is called quasi-analytic when for all real numbers $a<b$, the associated Denjoy-Carleman class

$$
\mathcal{C}_{\mathcal{M}}([a, b])=\left\{f \in \mathcal{C}^{\infty}([a, b], \mathbb{C}): \forall k \geq 0, \forall x \in[a, b],\left|f^{(k)}(x)\right| \leq M_{k}\right\}
$$

is quasi-analytic, meaning that any function belonging to this class is identically equal to zero when satisfying

$$
\exists x_{0} \in[a, b], \forall k \geq 0, \quad f^{(k)}\left(x_{0}\right)=0 .
$$

There exist several necessary and sufficient equivalent conditions that ensure a log-convex sequence to be quasianalytic. In this work, we will make use of the following one, which is a particular case of Denjoy-Carleman's theorem, see e.g. [13] (Thm. p. 91).

Theorem 4.1 (Denjoy-Carleman). A log-convex sequence $\left(M_{k}\right)_{k \geq 0}$ of positive real numbers is quasi-analytic if and only if

$$
\sum_{k=0}^{+\infty} \frac{M_{k}}{M_{k+1}}=+\infty
$$

For all connected open set $U \subset \mathbb{R}^{n}$, let us consider the more general Denjoy-Carleman class associated with a sequence $\mathcal{M}$ of positive real numbers:

$$
\mathcal{C}_{\mathcal{M}}(U)=\left\{f \in \mathcal{C}^{\infty}(U, \mathbb{C}): \forall \beta \in \mathbb{N}^{n},\left\|\partial_{x}^{\beta} f\right\|_{L^{\infty}(U)} \leq M_{|\beta|}\right\}
$$

We check in the following proposition that when the sequence $\mathcal{M}$ is quasi-analytic, then all the associated classes $C_{\mathcal{M}}(U)$ are also quasi-analytic.

Proposition 4.2. Let $U \subset \mathbb{R}^{n}$ be a connected open set and $\mathcal{M}$ be a quasi-analytic sequence of positive real numbers. Then, the associated class $C_{\mathcal{M}}(U)$ is quasi-analytic, meaning that any function $f$ belonging to this class that vanishes at infinite order in a point of the set $U$ is identically equal to zero.

Proof. This proof is based on elementary topology arguments. Let $f \in C_{\mathcal{M}}(U)$ vanishing at infinite order in a point of the set $U$, that is,

$$
\exists x_{0} \in U, \forall \beta \in \mathbb{N}^{n}, \quad \partial_{x}^{\beta} f\left(x_{0}\right)=0 .
$$


We set

$$
\Omega=\left\{x \in U: \forall \beta \in \mathbb{N}^{n}, \partial_{x}^{\beta} f(x)=0\right\} .
$$

The set $\Omega$ is non-empty and closed in $U$, since $f$ is a smooth function. Let us check that this is an open subset of $U$. Since $U$ is connected this will imply that $\Omega=U$ and therefore that the function $f$ is identically equal to zero. Let $x \in \Omega$. Since $U$ is open, there exists a radius $r>0$ such that $\overline{B(x, r)} \subset U$. Fixing $y \in B(x, r)$, we consider the function $g_{y}:[-2 r, 2 r] \rightarrow \mathbb{C}$ defined by

$$
g_{y}(t)=f\left(x+\frac{t(y-x)}{2 r}\right), \quad t \in[-2 r, 2 r] .
$$

Since $f \in C_{\mathcal{M}}(U)$, we get that $g_{y} \in C_{\mathcal{M}}([-2 r, 2 r])$. Indeed, it follows from a direct computation that for all $k \geq 0$ and $-2 r \leq t \leq 2 r$,

$$
\begin{aligned}
\left|g_{y}^{(k)}(t)\right| & =\left|\mathrm{d}^{k} f\left(x+\frac{t(y-x)}{2 r}\right) \cdot\left(\frac{y-x}{2 r}, \ldots, \frac{y-x}{2 r}\right)\right| \\
& \leq \sum_{1 \leq i_{1}, \ldots, i_{k} \leq n}\left|\frac{\partial^{k} f}{\partial_{x_{i_{1}}} \ldots \partial_{x_{i_{k}}}}\left(x+\frac{t(y-x)}{2 r}\right)\right|\left|\left(\frac{y-x}{2 r}\right)_{i_{1}}\right| \ldots\left|\left(\frac{y-x}{2 r}\right)_{i_{k}}\right| \\
& \leq\left(\sum_{1 \leq i_{1}, \ldots, i_{k} \leq n} \frac{1}{2^{i_{1}+\ldots+i_{k}}}\right) M_{k} \leq\left(\sum_{j=1}^{+\infty} \frac{1}{2^{j}}\right)^{k} M_{k}=M_{k} .
\end{aligned}
$$

Moreover, since $x \in \Omega$, we deduce from the definition (4.3) of the set $\Omega$ that

$$
\forall k \geq 0, \quad g_{y}^{(k)}(0)=0
$$

The sequence $\mathcal{M}$ being quasi-analytic by assumption, this implies that $g$ is identically equal to zero on $[-2 r, 2 r]$, and so $f(y)=g(2 r)=0$. The set $\Omega$ is then open in $U$.

We are now in position to state the main proposition of this subsection, which is instrumental in the proof of Theorem 2.6. This result is established in the work [10].

Proposition 4.3 (Corollary 2.8 in [10]). Let $\mathcal{M}$ be a log-convex quasi-analytic sequence, $t>0$ be a positive real number and $\omega \subset(0,1)^{n}$ be a measurable set. If $\operatorname{Leb}(\omega) \geq \lambda$ with $0<\lambda \leq 1$, then there exists a positive constant $C(\lambda, t, \mathcal{M}, n)>0$ such that for all $f \in \mathcal{C}_{\mathcal{M}}\left((0,1)^{n}\right)$ satisfying $\|f\|_{L^{\infty}\left((0,1)^{n}\right)} \geq t$,

$$
\|f\|_{L^{2}\left((0,1)^{n}\right)} \leq C(\lambda, t, \mathcal{M}, n)\|f\|_{L^{2}(\omega)} .
$$

A quantitative version of this result (with $L^{\infty}$ norms) is stated by Nazarov, Sodin and Volberg in [20] and this stronger result provides an explicit dependence of $C(\lambda, t, \mathcal{M}, n)$ with respect to $\lambda, t$ and $\mathcal{M}$ when $n=1$.

\subsection{Examples}

Now, we establish useful results to construct quasi-analytic sequences and we provide examples of such sequences. Let us begin with a straightforward general lemma.

Lemma 4.4. Let $\mathcal{M}$ and $\mathcal{M}^{\prime}$ be two sequences of positive real numbers satisfying $\mathcal{M} \leq \mathcal{M}^{\prime}$. Then, if $\mathcal{M}^{\prime}$ is a quasi-analytic sequence, so is the sequence $\mathcal{M}$. 
Proof. Since $\mathcal{M} \leq \mathcal{M}^{\prime}$ by assumption, we get that $C_{\mathcal{M}}([a, b]) \subset C_{\mathcal{M}^{\prime}}([a, b])$ for all real numbers $a<b$ and the result follows.

We now focus on the log-convex sequences of the form $\mathcal{M}^{F}$ defined in (4.2), which are the ones considered in this work. First, we study the stability of the quasi-analyticity property of the sequence $\mathcal{M}^{F}$ when modifying the function $F$.

Lemma 4.5. If the sequence $\mathcal{M}^{F}$ associated with a continuous function $F:[0,+\infty) \rightarrow \mathbb{R}$ bounded from below is quasi-analytic, then for all $c \in \mathbb{R}$ and $T>0$, the sequence $\mathcal{M}^{T F+c}$ associated with the continuous function $T F+c$ is also quasi-analytic.

Proof. By considering the function $G=F+c / T$, whose associated sequence $\mathcal{M}^{G}$ is immediately quasi-analytic, we can assume that $c=0$. Let us consider a positive integer $p \geq 1$ so that $T \geq 1 / p>0$. We therefore have that for all $k \geq 0$,

$$
\begin{aligned}
M_{k}^{T F}=\sup _{r \geq 0} r^{k} e^{-T F(r)} & \leq e^{\left(\frac{1}{p}-T\right) \inf F} \sup _{r \geq 0} r^{k} e^{-\frac{1}{p} F(r)} \\
& =e^{\left(\frac{1}{p}-T\right) \inf F}\left(\sup _{r \geq 0} r^{k p} e^{-F(r)}\right)^{\frac{1}{p}} \\
& =e^{\left(\frac{1}{p}-T\right) \inf F}\left(M_{k p}^{F}\right)^{\frac{1}{p}}=: M_{k} .
\end{aligned}
$$

According to Lemma 4.4, it is sufficient to check that the sequence $\left(M_{k}\right)_{k}$ is quasi-analytic to end the proof of Lemma 4.5. To that end, we will use Denjoy-Carleman's theorem, that is Theorem 4.1, and prove that

$$
\sum_{k=0}^{+\infty} \frac{M_{k}}{M_{k+1}}=+\infty
$$

Notice that since the sequence $\mathcal{M}^{F}$ is log-convex, the sequence $\left(M_{k}^{F} / M_{k+1}^{F}\right)_{k}$ is non-increasing. As a consequence, we get that

$$
\sum_{k=0}^{+\infty} \frac{M_{k}}{M_{k+1}}=\sum_{k=0}^{+\infty}\left(\frac{M_{k p}^{F}}{M_{k p+p}^{F}}\right)^{\frac{1}{p}}=\sum_{k=0}^{+\infty}\left(\prod_{j=0}^{p-1} \frac{M_{k p+j}^{F}}{M_{k p+j+1}^{F}}\right)^{\frac{1}{p}} \geq \sum_{k=0}^{+\infty} \frac{M_{k p+p-1}^{F}}{M_{k p+p}^{F}}
$$

It is therefore sufficient to prove that

$$
\sum_{k=0}^{+\infty} \frac{M_{k p+p-1}^{F}}{M_{k p+p}^{F}}=+\infty
$$

By using again that the sequence $\left(M_{k}^{F} / M_{k+1}^{F}\right)_{k}$ is non-increasing, we obtain by also applying an Euclidean division by the positive integer $p \geq 1$ that

$$
\sum_{k=0}^{+\infty} \frac{M_{k}^{F}}{M_{k+1}^{F}}=\sum_{r=0}^{p-1} \sum_{k=0}^{+\infty} \frac{M_{k p+r}^{F}}{M_{k p+r+1}^{F}} \leq p \sum_{k=0}^{+\infty} \frac{M_{k p}^{F}}{M_{k p+1}^{F}} .
$$

Yet, the log-convex sequence $\mathcal{M}^{F}$ is quasi-analytic by assumption, and Theorem 4.1 implies that

$$
\sum_{k=0}^{+\infty} \frac{M_{k}^{F}}{M_{k+1}^{F}}=+\infty \quad \text { and so } \quad \sum_{k=0}^{+\infty} \frac{M_{k p}^{F}}{M_{k p+1}^{F}}=+\infty .
$$


Finally, exploiting a last time the non-increasing property of the sequence $\left(M_{k}^{F} / M_{k+1}^{F}\right)_{k}$, we obtain that

$$
\sum_{k=0}^{+\infty} \frac{M_{(k+1) p}^{F}}{M_{(k+1) p+1}^{F}} \leq \sum_{k=0}^{+\infty} \frac{M_{(k+1) p-1}^{F}}{M_{(k+1) p}^{F}} .
$$

This inequality and (4.5) show that (4.4) holds. This ends the proof of Lemma 4.5.

It is a very interesting problem to characterize the functions $F$ that generate quasi-analytic sequences $\mathcal{M}^{F}$. This question has been addressed by B. Jaye and M. Mitkovski in [10], where these authors provided a necessary and sufficient condition on some functions $F$ that ensures the associated sequence $\mathcal{M}^{F}$ to be quasi-analytic, by exploiting Denjoy-Carleman's theorem.

Proposition $4.6([10])$. Let $F:[0,+\infty) \rightarrow \mathbb{R}$ be a function satisfying

(i) $F(0)=0$ and $F$ is non-decreasing with $\lim _{+\infty} F=+\infty$,

(ii) $F$ is lower-semicontinuous and $s \in \mathbb{R} \mapsto F\left(e^{s}\right)$ is convex.

Then, the sequence $\mathcal{M}^{F}$ associated with the function $F$ defined in (4.2) is quasi-analytic if and only if

$$
\int_{0}^{+\infty} \frac{F(t)}{1+t^{2}} \mathrm{~d} t=+\infty
$$

The following proposition provides some examples of functions $F$ generating quasi-analytic sequences $\mathcal{M}^{F}$. For the sake of completeness of the present work, we give a proof based only on Theorem 4.1, and not on Proposition 4.6.

Proposition 4.7. Let $p \geq 1$ be a positive integer and $F_{p}:[0,+\infty) \rightarrow[0,+\infty)$ be the non-negative function defined for all $t \geq 0$ by

$$
F_{p}(t)=\frac{t}{g(t)(g \circ g)(t) \ldots g^{\circ p}(t)}, \quad \text { where } \quad g(t)=\log (e+t)
$$

with $g^{\circ p}=g \circ \ldots \circ g$ (p compositions). The associated sequence $\mathcal{M}^{F_{p}}$ defined in (4.2) is a quasi-analytic sequence of positive real numbers.

Proof. We first notice that the sequence $\mathcal{M}^{F_{p}}$ is well-defined by (4.2) as the supremum of a continuous function on $[0,+\infty)$ with finite limit when $t \rightarrow+\infty$. Define $\phi_{p}:[0,+\infty) \rightarrow[0,+\infty)$ by

$$
\phi_{p}(t)=g(t)(g \circ g)(t) \ldots g^{\circ p}(t), \quad t \geq 0 .
$$

Let us check that the following convergence holds

$$
\frac{\phi_{p}(k)}{\phi_{p}\left(2 k \phi_{p}(k)\right)} \underset{k \rightarrow+\infty}{\rightarrow} 1
$$

Indeed, on the one hand, we get that

$$
\phi_{p}(k) \underset{k \rightarrow+\infty}{\sim}(\log k) \log (\log k) \ldots \log ^{\circ p} k
$$


On the other hand, we recall that if $f$ and $g$ are two numerical functions satisfying $f \sim_{+\infty} g$ with $\lim _{+\infty} f=+\infty$, then $\log f \sim_{+\infty} \log g$. As a consequence, we deduce that

$$
g\left(2 k \phi_{p}(k)\right) \underset{k \rightarrow+\infty}{\sim} \log \left(2 k(\log k) \log (\log k) \ldots \log ^{\circ p} k\right) \underset{k \rightarrow+\infty}{\sim} \log k .
$$

Iterating this argument, it follows that for all $j \geq 1$,

$$
g^{\circ j}\left(2 k \phi_{p}(k)\right) \underset{k \rightarrow+\infty}{\sim} \log ^{\circ j} k .
$$

This implies that the convergence (4.6) actually holds, since

$$
\frac{\phi_{p}(k)}{\phi_{p}\left(2 k \phi_{p}(k)\right)} \underset{k \rightarrow+\infty}{\sim} \frac{(\log k) \log (\log k) \ldots \log ^{\circ p} k}{(\log k) \log (\log k) \ldots \log ^{\circ p} k}=1
$$

Moreover, by direct computations, we have that for all $t \geq 0$,

$$
\begin{aligned}
\frac{\phi_{p}^{\prime}(t)}{\phi_{p}(t)} & =\sum_{i=1}^{p} \frac{\left(g^{\circ i}\right)^{\prime}(t)}{g^{\circ i}(t)}=\sum_{i=1}^{p} \frac{1}{g^{\circ i}(t)} \prod_{j=1}^{i} g^{\prime}\left(g^{\circ(j-1)}(t)\right) \\
& =\sum_{i=1}^{p} \frac{1}{g^{\circ i}(t)} \prod_{j=1}^{i} \frac{1}{e+g^{\circ(j-1)}(t)} \\
& =\frac{1}{e+t} \sum_{i=1}^{p} \frac{1}{g^{\circ i}(t)} \prod_{j=2}^{i} \frac{1}{e+g^{\circ(j-1)}(t)} \\
& ={ }_{t \rightarrow+\infty} o\left(\frac{1}{t}\right)
\end{aligned}
$$

that is

$$
\phi_{p}^{\prime}(t) \underset{t \rightarrow+\infty}{=} o\left(\frac{\phi_{p}(t)}{t}\right)
$$

For all positive integer $k \geq 1$, the supremum $M_{k}^{F_{p}}=\sup _{r \geq 0} r^{k} e^{-F_{p}(r)}$ is reached at a point $t_{k}>0$ satisfying the following equation

$$
t_{k} F_{p}^{\prime}\left(t_{k}\right)=k
$$

which is equivalent to

$$
\frac{t_{k}}{\phi_{p}\left(t_{k}\right)}\left(1-t_{k} \frac{\phi_{p}^{\prime}\left(t_{k}\right)}{\phi_{p}\left(t_{k}\right)}\right)=k
$$

Let us prove that $t_{k}$ exists and is unique for $k \gg 1$ sufficiently large. First, notice that the application $t \mapsto t F_{p}^{\prime}(t)$ satisfies $\lim _{t \rightarrow+\infty} t F_{p}^{\prime}(t)=+\infty$ since $\phi_{p}(t)={ }_{t \rightarrow+\infty} o(t)$ and that (4.8) holds. It remains to check that there exists 
$T>0$ such that this application is increasing on $[T,+\infty)$. To that end, it is sufficient to prove that the functions

$$
F_{p}: t \rightarrow \frac{t}{\phi_{p}(t)} \quad \text { and } \quad G_{p}: t \rightarrow 1-t \frac{\phi_{p}^{\prime}(t)}{\phi_{p}(t)}
$$

are positive and increasing on $[T,+\infty)$ for some $T \gg 1$ sufficiently large. The positivity of these functions is ensured by (4.8) and the positivity of $\phi_{p}$. Let us prove that $F_{p}$ and $G_{p}$ are increasing on $[T,+\infty)$ for $T$ sufficiently large. On the one hand, this fact holds true for the first function since

$$
F_{p}^{\prime}(t)=\frac{1}{\phi_{p}(t)}\left(1-t \frac{\phi_{p}^{\prime}(t)}{\phi_{p}(t)}\right) \underset{t \rightarrow+\infty}{\sim} \frac{1}{\phi_{p}(t)}>0
$$

On the other hand, it follows from (4.7) that for all $t \geq 0$,

$$
\begin{aligned}
-G_{p}^{\prime}(t) & =\left(\frac{t}{e+t} \sum_{i=1}^{p} \frac{1}{g^{\circ i}(t)} \prod_{j=2}^{i} \frac{1}{e+g^{\circ(j-1)}(t)}\right)^{\prime} \\
& =\frac{e}{(e+t)^{2}} \sum_{i=1}^{p} \frac{1}{g^{\circ i}(t)} \prod_{j=2}^{i} \frac{1}{e+g^{\circ(j-1)}(t)}+\frac{t}{e+t}\left(\sum_{i=1}^{p} \frac{1}{g^{\circ i}(t)} \prod_{j=2}^{i} \frac{1}{e+g^{\circ(j-1)}(t)}\right)^{\prime} .
\end{aligned}
$$

It can be checked that there exists a positive function $h_{2}$ given by a rational fraction of $g, g^{\circ 2}, \ldots, g^{\circ p}$ such that

$$
\left(\sum_{i=1}^{p} \frac{1}{g^{\circ i}(t)} \prod_{j=2}^{i} \frac{1}{e+g^{\circ(j-1)}(t)}\right)^{\prime}=-\frac{h_{2}(t)}{e+t} .
$$

We deduce from (4.10) and (4.11) that

$$
-G_{p}^{\prime}(t)=\frac{e}{(e+t)^{2}} h_{1}(t)-\frac{t}{(e+t)^{2}} h_{2}(t)=\frac{t}{(e+t)^{2}} h_{2}(t)\left(\frac{e}{t} \frac{h_{1}(t)}{h_{2}(t)}-1\right),
$$

with

$$
\forall t \geq 0, \quad h_{1}(t)=\sum_{i=1}^{p} \frac{1}{g^{\circ i}(t)} \prod_{j=2}^{i} \frac{1}{e+g^{\circ(j-1)}(t)}
$$

Since $h_{1} / h_{2}$ is a rational fraction of $g, g^{\circ 2}, \ldots, g^{\circ p}$, classical lemmas ensure that

$$
\frac{h_{1}(t)}{t h_{2}(t)} \underset{t \rightarrow+\infty}{\rightarrow} 0
$$

Therefore, since $h_{2}$ is positive, there exists a positive constant $T>0$ such that $G_{p}^{\prime}(t)>0$ for all $t \geq T$. It follows that for all $k \gg 1$ sufficiently large, there exists a unique $t_{k} \in(0,+\infty)$ satisfying (4.9). By defining $u_{k}=2 k \phi_{p}(k)$, it follows from (4.6) and (4.8) that

$$
u_{k} F_{p}^{\prime}\left(u_{k}\right)=\frac{u_{k}}{\phi_{p}\left(u_{k}\right)}\left(1-u_{k} \frac{\phi_{p}^{\prime}\left(u_{k}\right)}{\phi_{p}\left(u_{k}\right)}\right)=2 k \frac{\phi_{p}(k)}{\phi_{p}\left(2 k \phi_{p}(k)\right)}\left(1-u_{k} \frac{\phi_{p}^{\prime}\left(u_{k}\right)}{\phi_{p}\left(u_{k}\right)}\right) \underset{k \rightarrow+\infty}{\sim} 2 k .
$$


As a consequence, since $t_{k} F_{p}^{\prime}\left(t_{k}\right)=k$ from (4.9) and that the application $t \mapsto t F_{p}^{\prime}(t)$ is increasing on $[T,+\infty)$, we get that for all $k \gg 1$ sufficiently large,

$$
t_{k} \leq u_{k}=2 k \phi_{p}(k)
$$

We deduce that for all $k \gg 1$,

$$
\frac{M_{k-1}^{F_{p}}}{M_{k}^{F_{p}}} \geq \frac{t_{k}^{k-1} e^{-F_{p}\left(t_{k}\right)}}{t_{k}^{k} e^{-F_{p}\left(t_{k}\right)}}=\frac{1}{t_{k}} \geq \frac{1}{2 k \phi_{p}(k)} .
$$

It is well-known that the series $\sum \frac{1}{2 k \phi_{p}(k)}$ is divergent and then, we obtain

$$
\sum_{k=0}^{+\infty} \frac{M_{k}^{F_{p}}}{M_{k+1}^{F_{p}}}=+\infty
$$

Since the sequence $\mathcal{M}^{F_{p}}$ is log-convex by construction, we deduce from Theorem 4.1 that the sequence $\mathcal{M}^{F_{p}}$ is quasi-analytic. This ends the proof of Proposition 4.7.

Corollary 4.8. Let $s \geq 1,0 \leq \delta \leq 1$ be non-negative real numbers and $F_{s, \delta}:[0,+\infty) \rightarrow[0,+\infty)$ be the nonnegative continuous function defined by

$$
F_{s, \delta}(t)=\frac{t^{s}}{\log ^{\delta}(e+t)}, \quad t \geq 0
$$

The associated sequence $\mathcal{M}^{F_{s, \delta}}$ defined in (4.2) is a quasi-analytic sequence of positive real numbers.

Proof. Notice that there exists a positive constant $C_{s, \delta}>0$ such that for all $t \geq 0$,

$$
\frac{t^{s}}{\log ^{\delta}(e+t)} \geq \frac{t}{\log (e+t)}-C_{s, \delta}
$$

Corollary 4.8 is then a consequence of Lemma 4.4, Lemma 4.5 and Proposition 4.7.

\section{Proof of the APPRoximate NULL-CONTROLlability RESUlts}

This section is devoted to the proofs of the results stated in Section 2.2, dealing with the (cost-uniform) approximate null-controllability of the evolution equation

$$
\left\{\begin{array}{l}
\partial_{t} f(t, x)+F\left(\left|D_{x}\right|\right) f(t, x)=h(t, x) \mathbb{1}_{\omega}(x), \quad t>0, x \in \mathbb{R}^{n}, \\
f(0, \cdot)=f_{0} \in L^{2}\left(\mathbb{R}^{n}\right),
\end{array}\right.
$$

where the continuous function $F:[0,+\infty) \rightarrow \mathbb{R}$ bounded from below is assumed to be associated with a quasianalytic sequence $\mathcal{M}^{F}$ of positive real numbers, defined in (2.3), and $\omega \subset \mathbb{R}^{n}$ is a measurable set with positive Lebesgue measure.

\subsection{Proof of Proposition 2.10}

The purpose of this first subsection is to prove that the evolution equation $\left(E_{F}\right)$ is approximately nullcontrollable from the support $\omega$ in any positive time $T>0$ (with no extra assumption on $\omega$ ). It is well-known, see e.g. [6] (Thm. 2.43, page 56) or [21] (Rem. 16), that the approximate null-controllability (without uniform 
cost) of the system $\left(E_{F}\right)$ is equivalent to a unique continuation property of the adjoint system. More precisely, the adjoint system

$$
\left\{\begin{array}{l}
\partial_{t} g(t, x)+F\left(\left|D_{x}\right|\right) g(t, x)=0, \quad t>0, x \in \mathbb{R}^{n} \\
g(0, \cdot)=g_{0} \in L^{2}\left(\mathbb{R}^{n}\right)
\end{array}\right.
$$

is said to satisfy the unique continuation property from $\omega \subset \mathbb{R}^{n}$ at some time $T>0$ if for all initial datum $g_{0} \in L^{2}\left(\mathbb{R}^{n}\right)$, we have

$$
\left(\forall t \in[0, T], \mathbb{1}_{\omega} e^{-t F\left(\left|D_{x}\right|\right)} g_{0}=0\right) \Rightarrow\left(e^{-T F\left(\left|D_{x}\right|\right)} g_{0}=0\right)
$$

Let $T>0$ be a fixed positive time. We aim at proving that the unique continuation property holds from the control support $\omega$.

Let us first establish that for all $g \in L^{2}\left(\mathbb{R}^{n}\right)$, the function $e^{-T F\left(\left|D_{x}\right|\right)} g$ belongs to a class of quasi-analytic functions. We consider $g \in L^{2}\left(\mathbb{R}^{n}\right)$ not being identically equal to zero (the result is straightforward when $g=0$ ). Notice that for all $\beta \in \mathbb{N}^{n}$,

$$
\xi \rightarrow \xi^{\beta} \mathscr{F}\left(e^{-T F\left(\left|D_{x}\right|\right)} g\right) \in L^{1}\left(\mathbb{R}^{n}\right),
$$

since we have by Plancherel's theorem that

$$
\begin{aligned}
\int_{\mathbb{R}^{n}}\left|\xi^{\beta} \mathscr{F}\left(e^{-T F\left(\left|D_{x}\right|\right)} g\right)(\xi)\right| \mathrm{d} \xi & =\int_{\mathbb{R}^{n}}\left|\xi^{\beta} e^{-T F(|\xi|)} \widehat{g}(\xi)\right| \mathrm{d} \xi \\
& \leq(2 \pi)^{n / 2}\left(\int_{\mathbb{R}^{n}}|\xi|^{2|\beta|} e^{-2 T F(|\xi|)} \mathrm{d} \xi\right)^{\frac{1}{2}}\|g\|_{L^{2}\left(\mathbb{R}^{n}\right)} \\
& =(2 \pi)^{n / 2}\left(\int_{\mathbb{R}^{n}} \frac{\left(|\xi|^{2|\beta|}+|\xi|^{2|\beta|+2 n}\right) e^{-2 T F(|\xi|)}}{1+|\xi|^{2 n}} \mathrm{~d} \xi\right)^{\frac{1}{2}}\|g\|_{L^{2}\left(\mathbb{R}^{n}\right)} \\
& \leq C_{n}\left(\left(M_{|\beta|}^{T F}\right)^{2}+\left(M_{|\beta|+n}^{T F}\right)^{2}\right)^{\frac{1}{2}}\|g\|_{L^{2}\left(\mathbb{R}^{n}\right)} \\
& <+\infty
\end{aligned}
$$

the positive constant $C_{n}>0$ being given by

$$
C_{n}=(2 \pi)^{n / 2}\left(\int_{\mathbb{R}^{n}} \frac{1}{1+|\xi|^{2 n}} \mathrm{~d} \xi\right)^{\frac{1}{2}}<+\infty .
$$

Since the sequence $\mathcal{M}^{T F}$ is log-convex, there exists a positive constant $A_{T F}>0$ such that

$$
\forall k \geq 0, \quad M_{k}^{T F} \leq A_{T F} M_{k+1}^{T F}
$$

and we get from (5.1) that for all $\beta \in \mathbb{N}^{n}$,

$$
\int_{\mathbb{R}^{n}}\left|\xi^{\beta} \mathscr{F}\left(e^{-T F\left(\left|D_{x}\right|\right)} g\right)(\xi)\right| \mathrm{d} \xi \leq C_{n, T F} M_{|\beta|+n}^{T F}\|g\|_{L^{2}\left(\mathbb{R}^{n}\right)},
$$


with $C_{n, T F}=C_{n}\left(1+A_{T F}^{2 n}\right)^{\frac{1}{2}}$. It then follows from the Fourier inverse formula that

$$
\forall \beta \in \mathbb{N}^{n}, \quad \partial_{x}^{\beta}\left(e^{-T F\left(\left|D_{x}\right|\right)} g\right) \in L^{\infty}\left(\mathbb{R}^{n}\right),
$$

and that there exists a positive constant $C_{n, T F}^{\prime}>0$ such that

$$
\forall \beta \in \mathbb{N}^{n}, \quad\left\|\partial_{x}^{\beta}\left(e^{-T F\left(\left|D_{x}\right|\right)} g\right)\right\|_{L^{\infty}\left(\mathbb{R}^{n}\right)} \leq C_{n, T F}^{\prime} M_{|\beta|+n}^{T F}\|g\|_{L^{2}\left(\mathbb{R}^{n}\right)} .
$$

Notice that for all positive real number $\lambda>0$, the sequence $\mathcal{M}_{\lambda}$ whose elements $M_{\lambda, k}$ are given for all $k \geq 0$ by

$$
M_{\lambda, k}=C_{n, T F}^{\prime} \lambda^{k} M_{k+n}^{T F}\|g\|_{L^{2}\left(\mathbb{R}^{n}\right)},
$$

also defines a log-convex sequence which satisfies

$$
\sum_{k=0}^{+\infty} \frac{M_{\lambda, k}}{M_{\lambda, k+1}}=\frac{1}{\lambda} \sum_{k=n}^{+\infty} \frac{M_{k}^{T F}}{M_{k+1}^{T F}} .
$$

Since the sequence $\mathcal{M}^{F}$ is quasi-analytic, so is the sequence $\mathcal{M}^{T F}$ according to Lemma 4.5 and therefore, Theorem 4.1 implies that

$$
\sum_{k=0}^{+\infty} \frac{M_{k}^{T F}}{M_{k+1}^{T F}}=+\infty
$$

A second application of this theorem shows that the log-convex sequence $\mathcal{M}_{\lambda}$ is also quasi-analytic. This fact, combined with the estimate (5.2) and Proposition 4.2, implies that for all $g \in L^{2}\left(\mathbb{R}^{n}\right)$, the function $e^{-T F\left(\left|D_{x}\right|\right)} g$ belongs to the quasi-analytic class $\mathcal{C}_{\mathcal{M}_{1}}\left(\mathbb{R}^{n}\right)$.

Now, let us check that the unique continuation property holds. To that end, we make a weaker assumption by considering a function $g \in L^{2}\left(\mathbb{R}^{n}\right)$ not identically equal to zero such that

$$
\mathbb{1}_{\omega} e^{-T F\left(\left|D_{x}\right|\right)} g=0 .
$$

The purpose is to prove that $e^{-T F\left(\left|D_{x}\right|\right)} g=0$. Since $\operatorname{Leb}(\omega)>0$, if we consider the cube $Q=(-1,1)^{n}$, then

$$
\exists \lambda_{0}>0, \forall \lambda \geq \lambda_{0}, \quad \operatorname{Leb}(\omega \cap \lambda Q)>0 .
$$

Let us consider $\lambda \geq \lambda_{0}$ and define the function $g_{\lambda} \in L^{2}\left(\mathbb{R}^{n}\right)$ by

$$
g_{\lambda}(x)=\left(e^{-T F\left(\left|D_{x}\right|\right)} g\right)(\lambda x), \quad x \in \mathbb{R}^{n} .
$$

It readily follows from the estimate (5.2) that for all $\beta \in \mathbb{N}^{n}$,

$$
\left\|\partial_{x}^{\beta} g_{\lambda}\right\|_{L^{\infty}(Q)} \leq \lambda^{|\beta|}\left\|\partial_{x}^{\beta}\left(e^{-T F\left(\left|D_{x}\right|\right)} g\right)\right\|_{L^{\infty}\left(\mathbb{R}^{n}\right)} \leq C_{n, T F}^{\prime} \lambda^{|\beta|} M_{|\beta|+n}^{T F}\|g\|_{L^{2}\left(\mathbb{R}^{n}\right)} .
$$

Since the sequences $\mathcal{M}_{\lambda}$ defined in (5.3) are quasi-analytic and that $g_{\lambda} \in \mathcal{C}_{\mathcal{M}_{\lambda}}\left(\mathbb{R}^{n}\right)$ from the above estimate, Proposition 4.3 implies that for all $\lambda \geq \lambda_{0}$, there exists a positive constant $C=C\left(\lambda,\left\|g_{\lambda}\right\|_{L^{\infty}(Q)}, \omega, n\right)>0$ such that

$$
\left\|g_{\lambda}\right\|_{L^{2}(Q)} \leq C\left\|g_{\lambda}\right\|_{L^{2}\left(\left(\lambda^{-1} \omega\right) \cap Q\right)}
$$


As a consequence, we get from the assumption (5.4) that for all $\lambda \geq \lambda_{0}$,

$$
\left\|e^{-T F\left(\left|D_{x}\right|\right)} g\right\|_{L^{2}(\lambda Q)} \leq C\left\|e^{-T F\left(\left|D_{x}\right|\right)} g\right\|_{L^{2}(\omega \cap \lambda Q)}=0 .
$$

It follows that $\mathbb{1}_{\lambda Q} e^{-T F\left(\left|D_{x}\right|\right)} g=0$ for all $\lambda \geq \lambda_{0}$, and therefore $e^{-T F\left(\left|D_{x}\right|\right)} g=0$. Then, the unique continuation property holds and the evolution equation $\left(E_{F}\right)$ is approximately null-controllable from $\omega$ at time $T>0$.

\subsection{Proof of Theorem 2.6: sufficient part}

In this subsection, we aim at proving that when the support control $\omega$ is thick, then the equation $\left(E_{F}\right)$ is cost-uniformly approximately null-controllable from $\omega$ in any positive time $T>0$. To that end, we shall use the following observability characterization of the cost-uniform approximate null-controllability:

Theorem 5.1 (Proposition 6 in [21]). Let $T>0$ be a positive time. The following assertions are equivalent:

(i) The evolution system $\left(E_{F}\right)$ is cost-uniformly approximately null-controllable from $\omega$ in time $T>0$.

(ii) For all $\varepsilon \in(0,1)$, there exists a positive constant $C_{\varepsilon, T}>0$ such that for all $g \in L^{2}\left(\mathbb{R}^{n}\right)$,

$$
\left\|e^{-T F\left(\left|D_{x}\right|\right)} g\right\|_{L^{2}\left(\mathbb{R}^{n}\right)}^{2} \leq C_{\varepsilon, T} \int_{0}^{T}\left\|e^{-t F\left(\left|D_{x}\right|\right)} g\right\|_{L^{2}(\omega)}^{2} \mathrm{~d} t+\varepsilon\|g\|_{L^{2}\left(\mathbb{R}^{n}\right)}^{2} .
$$

According to Theorem 5.1, we need to prove that the observability estimate (5.5) holds. The first step consists in establishing the following uncertainty principle:

Proposition 5.2. For all time $T>0$ and for all $\varepsilon>0$, there exists a positive constant $C_{\varepsilon, T}>0$ such that for all $t \geq T / 2$ and $g \in L^{2}\left(\mathbb{R}^{n}\right)$,

$$
\left\|e^{-t F\left(\left|D_{x}\right|\right)} g\right\|_{L^{2}\left(\mathbb{R}^{n}\right)}^{2} \leq C_{\varepsilon, T}\left\|e^{-t F\left(\left|D_{x}\right|\right)} g\right\|_{L^{2}(\omega)}^{2}+\varepsilon e^{-2 t \inf F}\|g\|_{L^{2}\left(\mathbb{R}^{n}\right)}^{2} .
$$

Proof. This proof is adapted from the one of [10] (Thm. 1.3). Let $T>0$ and $\varepsilon>0$ fixed. Considering the function $G=F-\inf F$, we begin by proving that there exists a positive constant $C_{\varepsilon, T}>0$ such that for all $t \geq T / 2$ and $g \in L^{2}\left(\mathbb{R}^{n}\right)$,

$$
\left\|e^{-t G\left(\left|D_{x}\right|\right)} g\right\|_{L^{2}\left(\mathbb{R}^{n}\right)}^{2} \leq C_{\varepsilon, T}\left\|e^{-t G\left(\left|D_{x}\right|\right)} g\right\|_{L^{2}(\omega)}^{2}+\varepsilon\|g\|_{L^{2}\left(\mathbb{R}^{n}\right)}^{2} .
$$

Once the estimate (5.6) is established, we deduce that for all $t \geq T / 2$ and $g \in L^{2}\left(\mathbb{R}^{n}\right)$,

$$
\left\|e^{-t F\left(\left|D_{x}\right|\right)} g\right\|_{L^{2}\left(\mathbb{R}^{n}\right)}^{2} \leq C_{\varepsilon, T}\left\|e^{-t F\left(\left|D_{x}\right|\right)} g\right\|_{L^{2}(\omega)}^{2}+\varepsilon e^{-2 t \inf F}\|g\|_{L^{2}\left(\mathbb{R}^{n}\right)}^{2}
$$

and the proof of Proposition 5.2 is therefore ended.

Let us prove the estimate (5.6). Setting $T^{\prime}=T / 2$, we first check, using Parseval's formula, that the following Bernstein type estimates hold for all $g \in L^{2}\left(\mathbb{R}^{n}\right), t \geq T^{\prime}$ and $\beta \in \mathbb{N}^{n}$ :

$$
\begin{aligned}
& \left\|\partial_{x}^{\beta}\left(e^{-t G\left(\left|D_{x}\right|\right)} g\right)\right\|_{L^{2}\left(\mathbb{R}^{n}\right)}^{2}=\frac{1}{(2 \pi)^{n}} \int_{\mathbb{R}^{n}} e^{-2 t G(|\xi|)}\left|\xi^{\beta}\right|^{2}|\widehat{g}(\xi)|^{2} \mathrm{~d} \xi \\
& \leq \frac{1}{(2 \pi)^{n}} \int_{\mathbb{R}^{n}} e^{-2 T^{\prime} G(|\xi|)}|\xi|^{2|\beta|}|\widehat{g}(\xi)|^{2} \mathrm{~d} \xi \\
& \leq\left(\sup _{r \geq 0} r^{|\beta|} e^{-T^{\prime} G(r)}\right)^{2}\|g\|_{L^{2}\left(\mathbb{R}^{n}\right)}^{2}
\end{aligned}
$$




$$
=\left(M_{|\beta|}^{T^{\prime} G}\right)^{2}\|g\|_{L^{2}\left(\mathbb{R}^{n}\right)}^{2} .
$$

These estimates will be central later to control the functions $e^{-t G\left(\left|D_{x}\right|\right)} g$ on bad cubes, which we are about to define. Since $\omega$ is a thick set, by definition, there exist $\gamma \in(0,1]$ and a length $L>0$ such that

$$
\forall x \in \mathbb{R}^{n}, \quad \operatorname{Leb}\left(\omega \cap\left(x+[0, L]^{n}\right)\right) \geq \gamma L^{n}
$$

For $\alpha \in L \mathbb{Z}^{n}$, let us define $Q(\alpha)$ as the following cube

$$
Q(\alpha)=\alpha+[0, L]^{n}
$$

Notice that the family of cubes $(Q(\alpha))_{\alpha \in L \mathbb{Z}^{n}}$ covers the space $\mathbb{R}^{n}$ :

$$
\mathbb{R}^{n}=\bigcup_{\alpha \in L \mathbb{Z}^{n}} Q(\alpha)
$$

Let $g \in L^{2}\left(\mathbb{R}^{n}\right)$ and $t \geq T^{\prime}$, where we recall that $T^{\prime}=T / 2$. A cube $Q(\alpha)$ is said to be good if it satisfies the property

$$
\forall \beta \in \mathbb{N}^{n}, \quad\left\|\partial_{x}^{\beta}\left(e^{-t G\left(\left|D_{x}\right|\right)} g\right)\right\|_{L^{2}(Q(\alpha))}^{2} \leq \frac{2^{2|\beta|+n}}{\varepsilon}\left(M_{|\beta|}^{T^{\prime} G}\right)^{2}\left\|e^{-t G\left(\left|D_{x}\right|\right)} g\right\|_{L^{2}(Q(\alpha))}^{2},
$$

where the positive real numbers $M_{k}^{T^{\prime} G}$ are the elements of the sequence $\mathcal{M}^{T^{\prime} G}$ generated by the function $T^{\prime} G$. We recall that for all $k \geq 0$,

$$
M_{k}^{T^{\prime} G}=\sup _{r \geq 0} r^{k} e^{-T^{\prime} G(r)}
$$

Naturally, a cube $Q(\alpha)$ is said to be bad if it is not good, that is, when

$$
\exists \beta_{0} \in \mathbb{N}^{n}, \quad\left\|\partial_{x}^{\beta_{0}}\left(e^{-t G\left(\left|D_{x}\right|\right)} g\right)\right\|_{L^{2}(Q(\alpha))}^{2}>\frac{2^{2\left|\beta_{0}\right|+n}}{\varepsilon}\left(M_{\left|\beta_{0}\right|}^{T^{\prime} G}\right)^{2}\left\|e^{-t G\left(\left|D_{x}\right|\right)} g\right\|_{L^{2}(Q(\alpha))}^{2} .
$$

Notice from the covering property (5.8) that

$$
\left\|e^{-t G\left(\left|D_{x}\right|\right)} g\right\|_{L^{2}\left(\mathbb{R}^{n}\right)}^{2}=\sum_{\text {good cubes }}\left\|e^{-t G\left(\left|D_{x}\right|\right)} g\right\|_{L^{2}(Q(\alpha))}^{2}+\sum_{\text {bad cubes }}\left\|e^{-t G\left(\left|D_{x}\right|\right)} g\right\|_{L^{2}(Q(\alpha))}^{2} .
$$

We shall estimate independently the two terms of the right-hand side of this equality. Let us begin with the second one. It follows from the definition (5.10) that if $Q(\alpha)$ is a bad cube, there exists $\beta_{0} \in \mathbb{N}^{n}$ such that

$$
\begin{aligned}
\left\|e^{-t G\left(\left|D_{x}\right|\right)} g\right\|_{L^{2}(Q(\alpha))}^{2} & \leq \frac{\varepsilon}{2^{2\left|\beta_{0}\right|+n}\left(M_{\left|\beta_{0}\right|}^{T^{\prime} G}\right)^{2}}\left\|\partial_{x}^{\beta_{0}}\left(e^{-t G\left(\left|D_{x}\right|\right)} g\right)\right\|_{L^{2}(Q(\alpha))}^{2} \\
& \leq \sum_{\beta \in \mathbb{N}^{n}} \frac{\varepsilon}{2^{2|\beta|+n}\left(M_{|\beta|}^{T^{\prime} G}\right)^{2}}\left\|\partial_{x}^{\beta}\left(e^{-t G\left(\left|D_{x}\right|\right)} g\right)\right\|_{L^{2}(Q(\alpha))}^{2}
\end{aligned}
$$


By summing over all the bad cubes, we obtain from (5.7) and (5.12) that

$$
\begin{aligned}
\int_{\bigcup_{\text {bad cubes }}}\left|e^{-t G\left(\left|D_{x}\right|\right)} g(x)\right|^{2} \mathrm{~d} x & \leq \varepsilon \sum_{\text {bad cubes } \beta \in \mathbb{N}^{n}} \frac{1}{2^{2|\beta|+n}\left(M_{|\beta|}^{T^{\prime} G}\right)^{2}}\left\|\partial_{x}^{\beta}\left(e^{-t G\left(\left|D_{x}\right|\right)} g\right)\right\|_{L^{2}(Q(\alpha))}^{2} \\
& \leq \varepsilon \sum_{\beta \in \mathbb{N}^{n}} \frac{1}{2^{2|\beta|+n}\left(M_{|\beta|}^{T^{\prime} G}\right)^{2}}\left\|\partial_{x}^{\beta}\left(e^{-t G\left(\left|D_{x}\right|\right)} g\right)\right\|_{L^{2}\left(\mathbb{R}^{n}\right)}^{2} \\
& \leq \varepsilon \sum_{\beta \in \mathbb{N}^{n}} \frac{1}{2^{2|\beta|+n}}\|g\|_{L^{2}\left(\mathbb{R}^{n}\right)}^{2} \\
& \leq \varepsilon\|g\|_{L^{2}\left(\mathbb{R}^{n}\right)}^{2},
\end{aligned}
$$

since

$$
\sum_{\beta \in \mathbb{N}^{n}} \frac{1}{2^{2|\beta|+n}}=\sum_{k=0}^{+\infty}\left(\begin{array}{c}
k+n-1 \\
k
\end{array}\right) \frac{1}{2^{2 k+n}} \leq \sum_{k=0}^{+\infty} 2^{k+n-1} \frac{1}{2^{2 k+n}}=1
$$

It remains to estimate the first term of the right-hand side of the equality (5.11). Let $Q(\alpha)$ be a good cube. Assuming that the function $e^{-t G\left(\left|D_{x}\right|\right)} g$ is not identically equal to zero on the cube $Q(\alpha)$, we define the following function $\varphi:[0,1]^{n} \rightarrow \mathbb{C}$ by

$$
\varphi(z)=\frac{L^{\frac{n}{2}}\left(e^{-t G\left(\left|D_{x}\right|\right)} g\right)(\alpha+L z)}{\left\|e^{-t G\left(\left|D_{x}\right|\right)} g\right\|_{L^{2}(Q(\alpha))}}, \quad z \in[0,1]^{n} .
$$

As the cube $[0,1]^{n}$ satisfies the cone condition, the following Sobolev embedding holds

$$
W^{n, 2}\left([0,1]^{n}\right) \hookrightarrow L^{\infty}\left([0,1]^{n}\right),
$$

see e.g. [1] (Thm. 4.12). This implies that there exists a positive constant $C_{n}>0$, only depending on the dimension $n \geq 1$, such that

$$
\forall u \in W^{n, 2}\left([0,1]^{n}\right), \quad\|u\|_{L^{\infty}\left([0,1]^{n}\right)} \leq C_{n}\|u\|_{W^{n, 2}\left([0,1]^{n}\right)} .
$$

From the definition (5.9) of good cube and the estimate (5.15), it follows that for all $\beta \in \mathbb{N}^{n}$,

$$
\begin{aligned}
\left\|\partial_{x}^{\beta} \varphi\right\|_{L^{\infty}\left([0,1]^{n}\right)}^{2} & \leq C_{n}^{2} \sum_{\tilde{\beta} \in \mathbb{N}^{n},|\tilde{\beta}| \leq n}\left\|\partial_{x}^{\beta+\tilde{\beta}} \varphi\right\|_{L^{2}\left([0,1]^{n}\right)}^{2} \\
& =\frac{C_{n}^{2}}{\left\|e^{-t G\left(\left|D_{x}\right|\right)} g\right\|_{L^{2}(Q(\alpha))}^{2}} L^{2|\beta|} \sum_{\tilde{\beta} \in \mathbb{N}^{n},|\tilde{\beta}| \leq n} L^{2|\tilde{\beta}|}\left\|\partial_{x}^{\beta+\tilde{\beta}} e^{-t G\left(\left|D_{x}\right|\right)} g\right\|_{L^{2}(Q(\alpha))}^{2} \\
& \leq C_{n}^{2}(2 L)^{2|\beta|} 2^{n} \varepsilon^{-1} \sum_{\tilde{\beta} \in \mathbb{N}^{n},|\tilde{\beta}| \leq n}(2 L)^{2|\tilde{\beta}|}\left(M_{|\beta|+|\tilde{\beta}|}^{T^{\prime} G}\right)^{2} .
\end{aligned}
$$

Since the sequence $\mathcal{M}^{T^{\prime} G}$ is log-convex, there exists a positive constant $A_{T^{\prime} G}>0$ such that

$$
\forall k \geq 0, \quad M_{k}^{T^{\prime} G} \leq A_{T^{\prime} G} M_{k+1}^{T^{\prime} G} .
$$


Therefore, there exists a new positive constant $D_{n, L}>0$ depending on $n$ and $L$, such that for all $\beta \in \mathbb{N}^{n}$,

$$
\left\|\partial_{x}^{\beta} \varphi\right\|_{L^{\infty}\left([0,1]^{n}\right)}^{2} \leq \varepsilon^{-1} A_{T^{\prime} G}^{2 n} D_{n, L}^{2}(2 L)^{2|\beta|}\left(M_{|\beta|+n}^{T^{\prime} G}\right)^{2} .
$$

Let us recall that by assumption, the sequence $\mathcal{M}^{F}$ associated with the function $F$ defines a quasi-analytic sequence of positive real numbers, and so is $\mathcal{M}^{T^{\prime} G}$ according to Lemma 4.5. Notice that the slightly modified sequence $\mathcal{M}$, whose elements $M_{k}$ are given for all $k \geq 0$ by

$$
M_{k}=\varepsilon^{-1 / 2} A_{T^{\prime} G}^{n} D_{n, L}(2 L)^{k} M_{k+n}^{T^{\prime} G},
$$

also defines a log-convex sequence that satisfies

$$
\sum_{k=0}^{+\infty} \frac{M_{k}}{M_{k+1}}=\frac{1}{2 L} \sum_{k=n}^{+\infty} \frac{M_{k}^{T^{\prime} G}}{M_{k+1}^{T^{\prime} G}}
$$

Since the sequence $\mathcal{M}^{T^{\prime} G}$ is log-convex and quasi-analytic, Theorem 4.1 implies that

$$
\sum_{k=0}^{+\infty} \frac{M_{k}^{T^{\prime} G}}{M_{k+1}^{T^{\prime} G}}=+\infty
$$

and a second application of this theorem provides that the log-convex sequence $\mathcal{M}$ is also quasi-analytic. Since $\varphi \in \mathcal{C}_{\mathcal{M}}\left((0,1)^{n}\right)$, and that this function also satisfies

$$
\|\varphi\|_{L^{\infty}\left([0,1]^{n}\right)}=\frac{L^{\frac{n}{2}}\left\|e^{-t G\left(\left|D_{x}\right|\right)} g\right\|_{L^{\infty}(Q(\alpha))}}{\left\|e^{-t G\left(\left|D_{x}\right|\right)} g\right\|_{L^{2}(Q(\alpha))}} \geq 1,
$$

we therefore deduce from (5.17) and Proposition 4.3 that there exists a positive constant $C_{\varepsilon, \gamma, L, n, T, G}>0$ independent on $g$ and $\alpha$ such that

$$
\|\varphi\|_{L^{2}\left([0,1]^{n}\right)} \leq C_{\varepsilon, \gamma, L, n, T, G}\|\varphi\|_{L^{2}(E)},
$$

where $E=\frac{\omega-\alpha}{L} \cap[0,1]^{n} \subset[0,1]^{n}$ satisfies $\operatorname{Leb}(E) \geq \gamma>0$. It follows directly from (5.14) and (5.18) that

$$
\left\|e^{-t G\left(\left|D_{x}\right|\right)} g\right\|_{L^{2}(Q(\alpha))} \leq C_{\varepsilon, \gamma, L, n, T, G}\left\|e^{-t G\left(\left|D_{x}\right|\right)} g\right\|_{L^{2}(\omega \cap Q(\alpha))}
$$

Clearly, the above estimate also holds when the function $e^{-t G\left(\left|D_{x}\right|\right)} \mathrm{g}$ is identically equal to zero on the cube $Q(\alpha)$. By summing over all the good cubes, we deduce from (5.8) and (5.19) that

$$
\int_{\bigcup_{\text {good cubes }} Q(\alpha)}\left|e^{-t G\left(\left|D_{x}\right|\right)} g(x)\right|^{2} \mathrm{~d} x \leq C_{\varepsilon, \gamma, L, n, T, G}^{2} \int_{\omega}\left|e^{-t G\left(\left|D_{x}\right|\right)} g(x)\right|^{2} \mathrm{~d} x .
$$

This estimate, together with (5.11) and (5.13), imply that the estimate (5.6) actually holds. The proof of Proposition 5.2 is therefore ended.

We can now tackle the proof of the observability estimate (5.5). Let $\varepsilon>0$ and $T>0$. It follows from Proposition 5.2 that there exists a positive constant $C_{\varepsilon, T}>0$ such that for all $t \geq T / 2$ and $g \in L^{2}\left(\mathbb{R}^{n}\right)$,

$$
\left\|e^{-t F\left(\left|D_{x}\right|\right)} g\right\|_{L^{2}\left(\mathbb{R}^{n}\right)}^{2} \leq C_{\varepsilon, T}\left\|e^{-t F\left(\left|D_{x}\right|\right)} g\right\|_{L^{2}(\omega)}^{2}+\varepsilon^{\prime} e^{-2 t \inf F}\|g\|_{L^{2}\left(\mathbb{R}^{n}\right)}^{2},
$$


where we set

$$
\varepsilon^{\prime}=e^{2 T \inf F} \varepsilon
$$

By using that for all $s_{1} \geq s_{2} \geq 0$ and $g \in L^{2}\left(\mathbb{R}^{n}\right)$,

$$
\left\|e^{-s_{1} F\left(\left|D_{x}\right|\right)} g\right\|_{L^{2}\left(\mathbb{R}^{n}\right)} \leq e^{\left(s_{2}-s_{1}\right) \inf F}\left\|e^{-s_{2} F\left(\left|D_{x}\right|\right)} g\right\|_{L^{2}\left(\mathbb{R}^{n}\right)},
$$

it follows that for all $g \in L^{2}\left(\mathbb{R}^{n}\right)$,

$$
\begin{aligned}
& \left\|e^{-T F\left(\left|D_{x}\right|\right)} g\right\|_{L^{2}\left(\mathbb{R}^{n}\right)}^{2} \\
\leq & \frac{2}{T} \int_{\frac{T}{2}}^{T} e^{2(t-T) \inf F}\left\|e^{-t F\left(\left|D_{x}\right|\right)} g\right\|_{L^{2}\left(\mathbb{R}^{n}\right)}^{2} \mathrm{~d} t \\
\leq & \frac{2 C_{\varepsilon, T}}{T} \int_{\frac{T}{2}}^{T} e^{2(t-T) \inf F}\left\|e^{-t F\left(\left|D_{x}\right|\right)} g\right\|_{L^{2}(\omega)}^{2} \mathrm{~d} t+e^{-2 T \inf F} \varepsilon^{\prime}\|g\|_{L^{2}\left(\mathbb{R}^{n}\right)}^{2} \\
\leq & \frac{2 C_{\varepsilon, T}}{T} e^{-T(\inf F)_{-}} \int_{0}^{T}\left\|e^{-t F\left(\left|D_{x}\right|\right)} g\right\|_{L^{2}(\omega)}^{2} \mathrm{~d} t+\varepsilon\|g\|_{L^{2}\left(\mathbb{R}^{n}\right)}^{2},
\end{aligned}
$$

with $(\inf F)_{-}=\min (\inf F, 0)$. This ends the proof of the estimate (5.5).

\subsection{Cost-uniform approximate null-controllability vs rapid stabilization}

To end this section, let us quickly check that cost-uniform approximate null-controllability implies rapid stabilization:

Proposition 5.3. If the control system $\left(E_{F}\right)$ is cost-uniformly approximately null-controllable from $\omega$ at some positive time $T>0$, then is rapidly stabilizable from $\omega$.

Proof. Assume that the system $\left(E_{F}\right)$ is cost-uniformly approximately null-controllable from $\omega$ at some positive time $T>0$. According to Theorem 5.1, the following observability estimate holds: for all $\varepsilon>0$, there exists a positive constant $C_{\varepsilon, T}>0$ such that for all $g \in L^{2}\left(\mathbb{R}^{n}\right)$,

$$
\left\|e^{-T F\left(\left|D_{x}\right|\right)} g\right\|_{L^{2}\left(\mathbb{R}^{n}\right)}^{2} \leq C_{\varepsilon, T} \int_{0}^{T}\left\|e^{-t F\left(\left|D_{x}\right|\right)} g\right\|_{L^{2}(\omega)}^{2} \mathrm{~d} t+\varepsilon\|g\|_{L^{2}\left(\mathbb{R}^{n}\right)}^{2} .
$$

In order to prove that $\left(E_{F}\right)$ is rapidly stabilizable, it is sufficient to show that for all $\mu>0$, the system $\left(E_{F_{\mu}}\right)$ is stabilizable, where $F_{\mu}=F-\mu$. To that end, we shall apply Theorem 3.1, already used in Section 3.1. From (5.20), by multiplying by $e^{2 T \mu}$, we get that for all $\varepsilon>0$, there exists a positive constant $C_{\varepsilon, T}>0$ such that for all $g \in L^{2}\left(\mathbb{R}^{n}\right)$,

$$
\left\|e^{-T F_{\mu}\left(\left|D_{x}\right|\right)} g\right\|_{L^{2}\left(\mathbb{R}^{n}\right)}^{2} \leq C_{\varepsilon, T} \int_{0}^{T}\left\|e^{-t F_{\mu}\left(\left|D_{x}\right|\right)} g\right\|_{L^{2}(\omega)}^{2} \mathrm{~d} t+\varepsilon e^{2 T \mu}\|g\|_{L^{2}\left(\mathbb{R}^{n}\right)}^{2}
$$

By taking $\varepsilon=\frac{e^{-2 T \mu}}{2}$ in the above estimate, it follows from the characterization (iii) of Theorem 3.1 that the system $\left(E_{F_{\mu}}\right)$ is stabilizable from $\omega$. Since $\mu>0$ can be chosen arbitrary large, we deduce that the system $\left(E_{F}\right)$ is rapidly stabilizable from $\omega$. 


\section{APPENDIX A.}

This appendix is devoted to the proof of Proposition 3.3. The following arguments are due to H. Liu, G. Wang, Y. Xu and $\mathrm{H}$. Yu and are originally presented in [17] (Sect. 2). Let $F:[0,+\infty) \rightarrow \mathbb{R}$ be a continuous function bounded from below and $\omega \subset \mathbb{R}^{n}$ be a Borel set. Assume that the control system $\left(E_{F}\right)$ is stabilizable from $\omega$ at rate $\alpha>0$. We aim at proving that there exists a positive constant $A_{\alpha}>0$ such that for all $T>0$, there exists a positive constant $C_{\alpha, T}>0$ satisfying that for all $g \in L^{2}\left(\mathbb{R}^{n}\right)$,

$$
\left\|e^{-T F\left(\left|D_{x}\right|\right)} g\right\|_{L^{2}\left(\mathbb{R}^{n}\right)}^{2} \leq C_{\alpha, T} \int_{0}^{T}\left\|e^{-t F\left(\left|D_{x}\right|\right)} g\right\|_{L^{2}(\omega)}^{2} \mathrm{~d} t+A_{\alpha} e^{-2 \alpha T}\|g\|_{L^{2}\left(\mathbb{R}^{n}\right)}^{2} .
$$

By definition of stabilization, there exist a positive constant $M_{\alpha} \geq 1$ and a linear feedback $K_{\alpha} \in \mathcal{L}\left(L^{2}\left(\mathbb{R}^{n}\right)\right)$ such that

$$
\forall t \geq 0, \quad\left\|e^{-t\left(F\left(\left|D_{x}\right|\right)+\mathbb{1}_{\omega} K_{\alpha}\right)}\right\|_{\mathcal{L}\left(L^{2}\left(\mathbb{R}^{n}\right)\right)} \leq M_{\alpha} e^{-\alpha t}
$$

Let $T>0$ fixed. First notice from Duhamel's-type formula for bounded perturbations of semigroups, see e.g. [8] (Cor. III.1.7), that for all $f \in L^{2}\left(\mathbb{R}^{n}\right)$,

$$
e^{-T\left(F\left(\left|D_{x}\right|\right)+\mathbb{1}_{\omega} K_{\alpha}\right)} f=e^{-T F\left(\left|D_{x}\right|\right)} f-\int_{0}^{T} e^{-(T-t) F\left(\left|D_{x}\right|\right)} \mathbb{1}_{\omega} K_{\alpha} e^{-t\left(F\left(\left|D_{x}\right|\right)+\mathbb{1}_{\omega} K_{\alpha}\right)} f \mathrm{~d} t .
$$

By using the fact that the evolution operators $e^{-t F\left(\left|D_{x}\right|\right)}$ are selfadjoint on $L^{2}\left(\mathbb{R}^{n}\right)$, the above formula and (A.1) imply that for all $f, g \in L^{2}\left(\mathbb{R}^{n}\right)$,

$$
\begin{aligned}
& \left|\left\langle e^{-T F\left(\left|D_{x}\right|\right)} g, f\right\rangle_{L^{2}\left(\mathbb{R}^{n}\right)}\right|=\left|\left\langle g, e^{-T F\left(\left|D_{x}\right|\right)} f\right\rangle_{L^{2}\left(\mathbb{R}^{n}\right)}\right| \\
\leq & \left|\left\langle g, e^{-T\left(F\left(\left|D_{x}\right|\right)+\mathbb{1}_{\omega} K_{\alpha}\right)} f\right\rangle_{L^{2}\left(\mathbb{R}^{n}\right)}\right|+\left|\int_{0}^{T}\left\langle g, e^{-(T-t) F\left(\left|D_{x}\right|\right)} \mathbb{1}_{\omega} K_{\alpha} e^{-t\left(F\left(\left|D_{x}\right|\right)+\mathbb{1}_{\omega} K_{\alpha}\right)} f\right\rangle_{L^{2}\left(\mathbb{R}^{n}\right)} \mathrm{d} t\right| \\
\leq & \left|\left\langle g, e^{-T\left(F\left(\left|D_{x}\right|\right)+\mathbb{1}_{\omega} K_{\alpha}\right)} f\right\rangle_{L^{2}\left(\mathbb{R}^{n}\right)}\right|+\int_{0}^{T}\left|\left\langle\mathbb{1}_{\omega} e^{-(T-t) F\left(\left|D_{x}\right|\right)} g, K_{\alpha} e^{-t\left(F\left(\left|D_{x}\right|\right)+\mathbb{1}_{\omega} K_{\alpha}\right)} f\right\rangle_{L^{2}\left(\mathbb{R}^{n}\right)}\right| \mathrm{d} t \\
\leq & M_{\alpha} e^{-\alpha T}\|f\|_{L^{2}\left(\mathbb{R}^{n}\right)}\|g\|_{L^{2}\left(\mathbb{R}^{n}\right)}+M_{\alpha}\left\|K_{\alpha}\right\|_{\mathcal{L}\left(L^{2}\left(\mathbb{R}^{n}\right)\right)}\|f\|_{L^{2}\left(\mathbb{R}^{n}\right)} \int_{0}^{T}\left\|e^{-t F\left(\left|D_{x}\right|\right)} g\right\|_{L^{2}(\omega)} \mathrm{d} t .
\end{aligned}
$$

By applying the above inequality to $f=e^{-T F\left(\left|D_{x}\right|\right)} g$, it follows that for all $g \in L^{2}\left(\mathbb{R}^{n}\right)$,

$$
\left\|e^{-T F\left(\left|D_{x}\right|\right)} g\right\|_{L^{2}\left(\mathbb{R}^{n}\right)} \leq M_{\alpha}\left\|K_{\alpha}\right\|_{\mathcal{L}\left(L^{2}\left(\mathbb{R}^{n}\right)\right)} \int_{0}^{T}\left\|e^{-t F\left(\left|D_{x}\right|\right)} g\right\|_{L^{2}(\omega)} \mathrm{d} t+M_{\alpha} e^{-\alpha T}\|g\|_{L^{2}\left(\mathbb{R}^{n}\right)} .
$$

Finally, we deduce from Hölder's inequality and the classical convexity inequality

$$
\forall a, b>0, \quad(a+b)^{2} \leq 2\left(a^{2}+b^{2}\right),
$$

that for all $g \in L^{2}\left(\mathbb{R}^{n}\right)$,

$$
\left\|e^{-T F\left(\left|D_{x}\right|\right)} g\right\|_{L^{2}\left(\mathbb{R}^{n}\right)}^{2} \leq 2 M_{\alpha}^{2}\left\|K_{\alpha}\right\|_{\mathcal{L}\left(L^{2}\left(\mathbb{R}^{n}\right)\right)}^{2} T \int_{0}^{T}\left\|e^{-t F\left(\left|D_{x}\right|\right)} g\right\|_{L^{2}(\omega)}^{2} \mathrm{~d} t+2 M_{\alpha}^{2} e^{-2 \alpha T}\|g\|_{L^{2}\left(\mathbb{R}^{n}\right)}^{2}
$$

and this concludes the proof of Proposition 3.3. 


\section{REFERENCES}

[1] R.A. Adams and J.J.F. Fournier, Vol. 140 of Sobolev spaces. Second edition, Pure and Applied Mathematics (Amsterdam). Elsevier/Academic Press, Amsterdam (2003).

[2] P. Alphonse, Régularité des solutions et contrôlabilité d'équations d'évolution associées à des opérateurs non-autoadjoints. Ph.D Thesis, University of Rennes 1, France (2020).

[3] P. Alphonse and J. Bernier, Smoothing properties of fractional Ornstein-Uhlenbeck semigroups and null-controllability. Bull. Sci. Math. 165 (2020).

[4] K. Beauchard, M. Egidi and K. Pravda-Starov, Geometric conditions for the null-controllability of hypoelliptic quadratic parabolic equations with moving control supports. C. R. Math. Acad. Sci. Paris 358 (2020) 651-700.

[5] K. Beauchard, P. Jaming and K. Pravda-Starov, Spectral inequality for finite combinations of Hermite functions and nullcontrollability of hypoelliptic quadratic equations. To appear in Studia Math.. (2021)

[6] J.-M. Coron, Vol. 136 of Control and nonlinearity. Mathematical Surveys and Monographs. American Mathematical Society, Providence, RI (2007).

[7] M. Egidi and I. Veselić, Sharp geometric condition for null-controllability of the heat equation on $\mathbb{R}^{d}$ and consistent estimates on the control cost. Arch. Math. (Basel) 111 (2018) 85-99.

[8] K.-J. Engel and R. Nagel, One-parameter semigroups for linear evolution equations. Vol 194 of Graduate Texts in Mathematics. Springer-Verlag, New York (2000).

[9] S. Huang, G. Wang and M. Wang, Characterizations of stabilizable sets for some parabolic equations in $\mathbb{R}^{n}$. J. Differ. Equ. 272 (2021) 255-288.

[10] B. Jaye and M. Mitkovski, Quantitative uniqueness property for $L^{2}$ functions with fast decaying, or sparsely supported, Fourier transform. Int. Math. Res. Not. (2021) rnab075, https://doi.org/10.1093/imrn/rnab075

[11] A. Koenig, Lack of null-controllability for the fractional heat equation and related equations. SIAM J. Control Optim. $\mathbf{5 8}$ (2020) 3130-3160.

[12] A. Koenig, Contrôlabilité de quelques équations aux dérivées partielles paraboliques peu diffusives. Ph.D thesis, University Côte d'Azur, France (2019).

[13] P. Koosis, The Logarithmic Integral I. Cambridge University Press, 1988.

[14] O. Kovrijkine, Some results related to the Logvinenko-Sereda theorem. Proc. Amer. Math. Soc. 129 (2001) $3037-3047$.

[15] G. Lebeau and L. Robbiano, Contrôle exact de l'équation de la chaleur. Comm. Partial Differ. Equ. 20 (1995) $335-356$.

[16] P. Lissy, A non-controllability result for the half-heat equation on the whole line based on the prolate spheroidal wave functions and its application to the Grushin equation. Preprint arXiv e-print (2020).

[17] H. Liu, G. Wang, Y. Xu and H. Yu, Characterizations on complete stabilizability. Preprint arXiv e-print (2020).

[18] J. Martin and K. Pravda-Starov, Geometric conditions for the exact controllability of fractional free and harmonic Schrödinger equations. J. Evol. Equ. 21 (2021) 1059-1087.

[19] L. Miller, A direct Lebeau-Robbiano strategy for the observability of heat-like semigroups. Discrete Contin. Dyn. Syst. Ser. B 14 (2010) 1465-1485.

[20] F. Nazarov, M. Sodin and A. Volberg, Lower bounds for quasianalytic functions, I. How to control smooth functions. Math. Scand. 95 (2004) 59-79.

[21] E. Trélat, G. Wang and Y. Xu, Characterization by observability inequalities of controllability and stabilization properties. Pure Appl. Anal. 2 (2020) 93-122.

[22] G. Wang, M. Wang, C. Zhang and Y. Zhang, Observable set, observability, interpolation inequality and spectral inequality for the heat equation in $\mathbb{R}^{n}$. J. Math. Pures Appl. 126 (2019) 144-194.

[23] S. Xiang, Quantitative rapid and finite time stabilization of the heat equation. Preprint arXiv e-print (2020).

\section{Subscribe to Open (S2O) A fair and sustainable open access model}

This journal is currently published in open access under a Subscribe-to-Open model (S2O). S2O is a transformative model that aims to move subscription journals to open access. Open access is the free, immediate, online availability of research articles combined with the rights to use these articles fully in the digital environment. We are thankful to our subscribers and sponsors for making it possible to publish this journal in open access, free of charge for authors.

\section{Please help to maintain this journal in open access!}

Check that your library subscribes to the journal, or make a personal donation to the S2O programme, by contacting subscribers@edpsciences.org

More information, including a list of sponsors and a financial transparency report, available at: https://www.edpsciences.org/en/maths-s2o-programme 